\title{
The age of blue LSB galaxies
}

\author{
E. I. Vorobyov ${ }^{1,2}$, Yu. Shchekinov ${ }^{5, \star}$, D. Bizyaev ${ }^{3,4}$, D. Bomans ${ }^{5}$, and R.-J. Dettmar ${ }^{5}$ \\ 1 The Institute for Computational Astrophysics, Saint Mary's University, Halifax NS, B3H 3C3, Canada \\ 2 Institute of Physics, South Federal University, Stachki 194, Rostov-on-Don, Russia \\ e-mail: eduard_vorobev@mail.ru \\ 3 New Mexico State University/APO, Sunspot, NM, 88349, USA \\ 4 Sternberg Astronomical Institute, Universitetsky 13, Moscow 119992, Russia \\ 5 Astronomisches Institut, Ruhr-Universität Bochum, Bochum, Germany
}

Received 3 June 2009 / Accepted 15 June 2009

\begin{abstract}
Context. Low metallicities, high gas-to-star mass ratios, and blue colors of most low surface brightness (LSB) galaxies imply that these systems may be younger than their high surface brightness counterparts.

Aims. We look for observational signatures that can help to constrain the age of blue LSB galaxies.

Methods. We use numerical hydrodynamic modelling to study the long-term ( 13 Gyr) dynamical and chemical evolution of blue LSB galaxies, adopting a sporadic scenario of star formation. Our model galaxy consists of a thin gas disk, a stellar disk with a developed spiral structure and a spherical dark matter halo. Our models utilize various rates of star formation and different shapes of the initial mass function (IMF). We complement hydrodynamic modelling with population synthesis modelling to produce the integrated $B-V$ colors and $\mathrm{H} \alpha$ equivalent widths.

Results. We find that the mean oxygen abundances, $B-V$ colors, $\mathrm{H} \alpha$ equivalent widths, and the radial fluctuations in the oxygen abundance, when considered altogether, can be used to constrain the age of blue LSB galaxies if some independent knowledge of the IMF is available. Our modelling strongly suggests the existence of a minimum age for blue LSB galaxies. Model $B-V$ colors and mean oxygen abundances set a tentative minimum age at 1.5-3.0 Gyr, whereas model $\mathrm{H} \alpha$ equivalent widths suggest a larger value of the order of 5-6 Gyr. The latter value may decrease somewhat if blue LSB galaxies host IMFs with a truncated upper mass limit. We found no firm evidence that the age of blue LSB galaxies is significantly lower than $13 \mathrm{Gyr}$.

Conclusions. The age of blue LSB galaxies may vary between 1.5-6 Gyr and $13 \mathrm{Gyr}$, depending on the physical conditions in the disk that control the form of the IMF and the rate of star formation. A failure to observationally detect large radial fluctuations in the oxygen abundance of the order of 0.5-1.0 dex, which, according to our modelling, are characteristic of (1-2)-Gyr-old galaxies, will argue in favour of the more evolved nature of blue LSB galaxies.
\end{abstract}

Key words. ISM: abundances - galaxies: abundances - galaxies: evolution

\section{Introduction}

Low surface brightness (LSB) galaxies have a central surface brightness much fainter than the Freeman value $\mu_{\mathrm{B}}=21.65 \pm$ $0.30 \mathrm{mag} \operatorname{arcsec}^{-2}$. These galaxies are thought to represent a significant fraction of the galaxy number density in the universe (McGaugh et al. 1995a; O’Neil \& Bothun 2000; Trachternach et al. 2006). LSB galaxies show a wide spread of colours ranging from red $(B-V \simeq 1.7)$ to very blue $(B-V \simeq 0.2)$ (O'Neil et al. 1997), but the most common type seems to be blue LSB galaxies: late-type, disk dominated spirals with central surface brightness $\mu_{\mathrm{B}} \gtrsim 23 \mathrm{mag} \operatorname{arcsec}^{-2}$ and colors lying in the range $(B-V)=0.3-0.7$. These blue LSB galaxies are among the most gas-rich (up to $50 \%$ of the total baryonic mass) and metal-deficient (about 5-20\% of the solar metallicity) galaxies (McGaugh 1994; Rönnback \& Bergvall 1995; McGaugh \& Blok 1997; de Blok \& van der Hulst 1998; Kuzio de Narray et al. 2004).

Low metallicities and large gas-to-star mass ratios may be interpreted in various ways. For instance, blue LSB galaxies may have formed recently, at redshifts close to $z=0$. However, this

\footnotetext{
^ On leave from Department of Physics, South Federal University, and Special Astrophysical Observatory.
}

can contradict the hierarchical scenario of large-scale structure formation, within which low-mass galaxies form first at redshifts close to the onset of the reionization epoch, $z \sim 10$. Therefore, it is more likely that blue LSB galaxies have formed at large $z$ but have had a delayed onset of the major star formation epoch. In this case, conflict with the hierarchical structure formation is relaxed. Indeed, blue LSB galaxies show a wide range of masses with an excess of dwarf systems with luminosities of the order of $10^{7}-10^{8} L_{\odot}$ (Sprayberry et al. 1997). Such dwarf systems are known to have a younger mean stellar age than their more massive counterparts - a phenomenon known as "downsizing" (e.g. Thomas et al. 2005). Another possible interpretation of the apparent unevolved nature of blue LSBs is that they do not form late, nor have a delayed onset of star formation, but simply evolve slower than their high surface brightness counterparts (e.g. van den Hoek et al. 2000).

There have been many efforts to infer the age of blue LSB galaxies from the properties of stellar populations. Broadband photometric studies, complemented by $\mathrm{H} \alpha$ emission line data and synthetic stellar population code modeling, predict quite a wide range for the ages of blue LSB galaxies: from 1-2 Gyr (Zackrisson et al. 2005) to 7-9 Gyr (Padoan et al. 1997; Jimenez et al. 1998). Rather young ages of blue LSB galaxies (<5 Gyr) 
follow also from their $(V-I)$ colors and HI content (Schombert et al. 2001). The comparison of measured spectral energy distributions in a sample of LSB galaxies with synthetic spectra (derived assuming an exponentially declining SFR) by Haberzettl et al. (2005) suggests the ages of the dominant stellar population to be between 2 and 5 Gyr.

In this paper, we perform numerical hydrodynamic modelling of the dynamical, chemical, and photometric evolution of blue LSB galaxies to constrain their typical range of ages. The cornerstone supposition of our modelling, motivated by observational evidence, is the sporadic nature of star formation in blue LSB galaxies. Indeed, McGaugh (1994), de Blok et al. (1995), Gerretsen \& de Blok (1999), and others argue that the primary cause of the blue colors is the young age of the dominant stellar population. This can take the form of young local bursts of star formation superimposed (or not) on a continuous low-rate star formation. Available $\mathrm{H} \alpha$ images of LSB galaxies (see e.g. McGaugh \& Bothun 1994; McGaugh et al. 1995b; Auld et al. 2006) appear to support this so-called sporadic star formation scenario. Current star formation is localized to a handful of compact regions. There is little or no diffuse $\mathrm{H} \alpha$ emission coming from the rest of the galactic disk. Randomly distributed star formation sites, along with inefficient spatial stirring of heavy elements, give rise to radial fluctuations in the oxygen abundance on spatial scales of the order of 1-2 kpc. The resulting fluctuation spectrum is age-sensitive and can be potentially used to set order-of-magnitude constraints on the age of blue LSB galaxies. However, the age prediction may be considerably improved by using the mean oxygen abundances and $\mathrm{H} \alpha$ equivalent widths provided that some independent knowledge of the initial mass function is available.

This paper is organized as follows. In the next section we discuss qualitatively the processes that mix heavy elements in disks of LSB galaxies. Section 3 details our theoretical model, while Sect. 4 describes our observations. In Sects. 5 and 6 we discuss our numerical results, and Sect. 7 presents our main conclusions.

\section{Mixing of heavy elements in LSB disks}

In high surface brightness (HSB) galaxies, mixing of heavy elements is provided by multiple shock waves from supernova explosions. It requires approximately $100 \mathrm{Myr}$ to homogenize heavy elements in the warm neutral interstellar medium of HSB galaxies (de Avillez \& MacLow 2002). LSB galaxies, on the other hand, are characterized by a star formation rate $\left(\sim 0.1 M_{\odot} \mathrm{yr}^{-1}\right)$ that is almost two orders of magnitude lower than the Milky Way value, $\approx 5 M_{\odot} \mathrm{yr}^{-1}$. Consequently, the frequency of shock waves in the interstellar medium of LSB galaxies and the corresponding volume filling factor occupied by the shock processed gas can be as small as $\Psi_{\text {sh }} \lesssim\left(200 \mathrm{Myr}^{-1}\right.$ and $f_{\text {sh }} \lesssim 0.004$, respectively. For comparison, the corresponding values in the Milky Way are $\Psi_{\text {sh }} \sim(5 \mathrm{Myr})^{-1}$ (Draine \& Salpeter 1979) and $f_{\mathrm{sh}} \sim 0.2$ (de Avillez \& Breitschwerdt 2005). In these conditions, homogenization of heavy elements in LSB galaxies may require a considerably longer time scale than $100 \mathrm{Myr}$, a typical value for HSB galaxies. Differential rotation of the galactic disk and radial convection driven by the spiral stellar density waves may in principle be efficient in mixing of heavy elements in LSB galaxies. However, the time scales for these processes in LSB galaxies are not well known and numerical simulations are needed to determine their importance. Therefore, the distribution of heavy elements in the disk of an LSB galaxy may be characterized by radial variations, the typical amplitude of which is expected to diminish with time. It is our purpose to determine whether these radial abundance fluctuations can be used to constrain the ages of LSB galaxies.

We note that mixing in the azimuthal direction is strongly enhanced by differential rotation. The size of a mixing eddy grows with time due to differential rotation as $\Delta x \sim\left(u_{\phi}\right)_{\mathrm{r}}^{\prime} D^{1 / 2} t^{3 / 2}$, much faster than due to hydrodynamic mixing $\Delta x \propto t$ (Scalo $\&$ Elmegreen 2004), where $\left(u_{\phi}\right)_{\mathrm{r}}^{\prime}$ is the radial derivative of the rotation velocity, $D$ is the diffusion coefficient. Differential rotation therefore homogenizes metals in the azimuthal direction faster than in the radial one and the radial variations remain for a longer time.

\section{Model LSB galaxy}

\subsection{Initial configuration}

LSB galaxies show a wide range of morphological types, ranging from giant spirals to dwarf irregular galaxies, from earlytype red galaxies to late-type blue ones. In this paper we focus on late-type blue LSB galaxies. According to the observational data (van den Hoek et al. 2000), a typical blue LSB galaxy has a dynamical mass of the order of $10^{10} M_{\odot}$ and HI mass of the order of $2 \times 10^{9} M_{\odot}$. We closely follow these estimates when constructing our model galaxy. Our model LSB galaxy consists of a thin gas disk, which evolves in the external gravitational potential of both the spherical dark matter halo and extended stellar disk. LSB galaxies often feature a few ill-defined spiral arms. Therefore, we assume that our model stellar disk has developed a two-armed spiral structure. Below, we provide a more detailed explanation for each of the constituents of our model galaxy.

The velocity field of most LSB galaxies is better fitted by dark matter models described by a modified isothermal sphere rather than by a NFW profile (Kuzio de Naray et al. 2006). Therefore, the spherical dark matter halo in our model is modelled by a radial density profile described by the modified isothermal sphere

$\rho_{\mathrm{h}}=\frac{\rho_{\mathrm{h} 0}}{1+\left(r / r_{\mathrm{h}}\right)^{2}}$,

where $\rho_{\mathrm{h} 0}=6.0 \times 10^{-3} M_{\odot} \mathrm{pc}^{-3}$ and $r_{\mathrm{h}}=5.7 \mathrm{kpc}$ are the central volume density and characteristic scale length of the dark matter halo, respectively, and $r$ is the radial distance. The adopted values of $\rho_{\mathrm{h} 0}$ and $r_{\mathrm{h}}$ are similar to those obtained by Kuzio de Naray et al. (2006) for UGC 5750. The dark matter halo mass in the inner $15 \mathrm{kpc}$ is approximately $2.0 \times 10^{10} M_{\odot}$. The radial gravity force (per unit mass) due to the spherical dark matter halo in the plane of the disk can be written as

$\frac{\partial \Phi_{\mathrm{h}}}{\partial r}=4 \pi G \rho_{\mathrm{h} 0} r_{\mathrm{h}}\left[r / r_{\mathrm{h}}-\arctan \left(r / r_{\mathrm{h}}\right)\right]\left(\frac{r_{\mathrm{h}}}{r}\right)^{2}$.

The stellar component of our model galaxy consists of two parts - an axisymmetric stellar disk and a two-armed spiral pattern. The former is described by a radially declining exponential profile

$\Sigma_{\mathrm{st}}=\Sigma_{\mathrm{s} 0} \exp \left(-r / r_{\mathrm{s}}\right)$,

where $\Sigma_{\mathrm{s} 0}=30 M_{\odot} \mathrm{pc}^{-2}$ is the central stellar surface density and $r_{\mathrm{s}}=4 \mathrm{kpc}$ is the radial scale length of the stellar disk. The adopted value for $r_{\mathrm{s}}$ is typical for blue LSB galaxies (de Blok et al. 1995). We note that our model stellar disk serves as a proxy for the real stellar disk that is expected to form during the evolution of our model galaxy. The mass of the stellar disk in the 
inner $15 \mathrm{kpc}$ is $M_{\mathrm{s}}=2.6 \times 10^{9} M_{\odot}$. For numerical purposes, the gravitational potential of the axisymmetric thin stellar disk in the plane of the disk is calculated as

$\Phi_{\mathrm{st}}(r, z=0)=-\pi G \Sigma_{\mathrm{s} 0} r\left[I_{0}(y) K_{1}(y)-I_{1}(y) K_{0}(y)\right]$,

where $I_{n}$ and $K_{n}$ are modified Bessel functions of the first and second kinds, respectively, and $y \equiv r /\left(2 r_{\mathrm{s}}\right)$.

The non-axisymmetric gravitational potential of stellar spiral arms is described in polar coordinates $(r, \phi)$ by a running density wave (see e.g. Vorobyov \& Shchekinov 2005)

$\Phi_{\mathrm{sp}}(r, \phi)=-C(r) \cos \left[m\left(\cot (i) \ln \left(r / r_{\mathrm{sp}}\right)+\phi-\Omega_{\mathrm{sp}} t\right)\right]$,

where $C(r)$ is the radially varying amplitude, $i$ is the pitch angle, $r_{\mathrm{sp}}$ is the characteristic radius of the spiral at $\phi=0, m=2$ is the number of spiral arms, and $\Omega_{\mathrm{sp}}$ is the angular velocity of the spiral pattern. In the following we adopt $r_{\mathrm{sp}}=6 \mathrm{kpc}$ and $\Omega_{\mathrm{sp}}=5.5 \mathrm{~km} \mathrm{~s}^{-1} \mathrm{kpc}$. The pitch angle is set to $25^{\circ}$, typical for the late-type spiral galaxies.

The amplitude $C(r)$ of the spiral stellar gravitational potential $\Phi_{\mathrm{sp}}$ determines the response of the gas and consequently the appearance of a spiral pattern in the gas disk. We adopt the following expression for the amplitude $C(r)=C_{0}(r)^{\alpha(r)}$. Here, $C_{0}(r)$ is a linear function of radius which has a value of 0 at $r=0 \mathrm{kpc}$ and equals $1.8 \times 10^{-3}$ (in dimensionless units) at $r=17 \mathrm{kpc}$. The amplitude is set to zero at the galactic center to ensure that the gravitational potential of the stellar spiral density wave diminishes at small galactocentric distances. Otherwise, even a small-amplitude spiral gravitational potential would produce strong azimuthal gravitational forces near the galactic center due to converging radial gridlines in polar coordinates, which quickly destroys the gas disk during the numerical simulations. The exponent $\alpha(r)$ decreases linearly with radius $r$. More specifically, we choose $\alpha(r=0 \mathrm{kpc})=2.3$, and $\alpha(r=17 \mathrm{kpc})=0.6$. This specific form of $C(r)$ allows us to produce spiral gravitational potentials with different shapes and maximum amplitudes. The resulting ratio $\beta(r)$ of the maximum non-axisymmetric gravitational acceleration due to spiral arms $\left[\left(\partial \Phi_{\mathrm{sp}} / \partial r\right)^{2}+\left(r^{-1} \partial \Phi_{\mathrm{sp}} / \partial \phi\right)^{2}\right]^{1 / 2}$ (at a given radial distance $r$ ) versus the gravitational acceleration due to both the dark matter halo $\partial \Phi_{\mathrm{h}} / \partial r$ and axisymmetric stellar disk $\partial \Phi_{\mathrm{st}} / \partial r$ is shown in Fig. 1 by the solid line. We note that $\beta$ never exceeds $19 \%$.

The measurements of Kuzio de Naray et al. (2006) indicate that the gas velocity dispersions of LSB galaxies lie in the range between $6 \mathrm{~km} \mathrm{~s}^{-1}$ and $10 \mathrm{~km} \mathrm{~s}^{-1}$ and are consistent with the typical dispersions for the gas component of HSB galaxies. Therefore, we assume that the gas disk of our model galaxy is initially isothermal at a temperature $T=10^{4} \mathrm{~K}$. LSB galaxies have same atomic hydrogen content as their HSB counterparts but generally lower surface densities and extended gas disks (Pickering 1999; de Blok et al. 1996). We assume that the gas disk has an exponentially declining density profile

$\Sigma_{\mathrm{g}}=\Sigma_{\mathrm{g} 0} \exp \left(-r / r_{\mathrm{g}}\right)$,

with the central surface density $\Sigma_{\mathrm{g} 0}=6.5 \mathrm{M}_{\odot} \mathrm{pc}^{-2}$ and radial scale length $r_{\mathrm{g}}=30 \mathrm{kpc}$. The gas disk extends to $17 \mathrm{kpc}$ in our model, but star formation is confined to the inner $15 \mathrm{kpc}$. The outermost $2 \mathrm{kpc}$ are kept for numerical reasons. Gas disks of such an extent $(\sim 15 \mathrm{kpc})$ are typical for LSB galaxies (van den Hoek et al. 2000). The mean surface density of our model disk is $\left\langle\Sigma_{\mathrm{g}}\right\rangle=4.9 M_{\odot} \mathrm{pc}^{-2}$, which is comparable to a median HI surface density $\Sigma_{\mathrm{HI}}=3.8 M_{\odot} \mathrm{pc}^{-2}$ of a sample of LSB galaxies in van den Hoek et al. (2000). The mass of our model

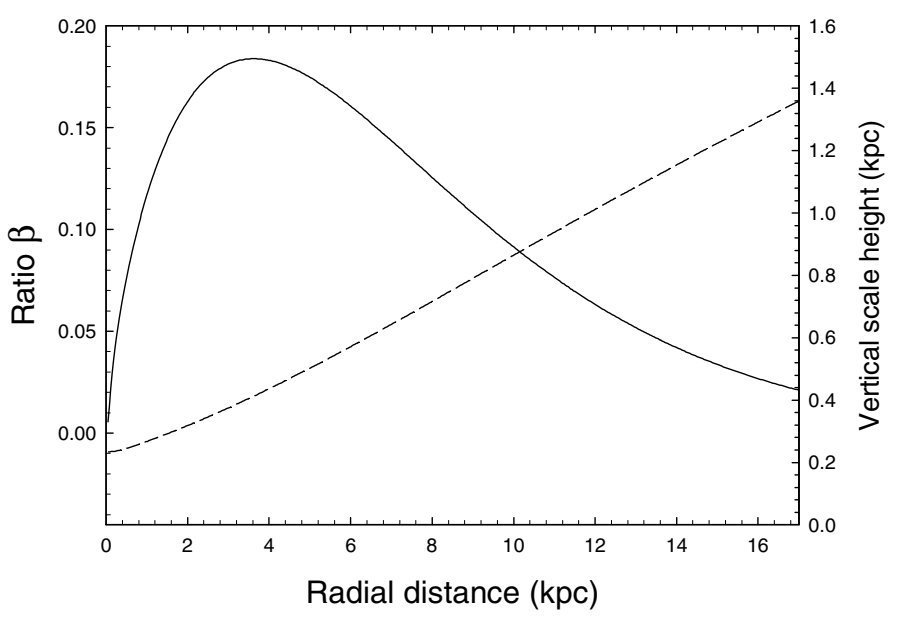

Fig. 1. (Solid line) Ratio $\beta(r)$ of the maximum non-axisymmetric gravitational acceleration due to spiral arms $\left[\left(\partial \Phi_{\mathrm{sp}} / \partial r\right)^{2}+\left(r^{-1} \partial \Phi_{\mathrm{sp}} / \partial \phi\right)^{2}\right]^{1 / 2}$ vesus gravitational acceleration due to both the dark matter halo $\partial \Phi_{\mathrm{h}} / \partial r$ and axisymmetric stellar disk $\partial \Phi_{\mathrm{st}} / \partial r$. (Dashed line) Vertical scale height $Z$ of our model gas disk as a function of radius at the beginning of numerical simulations $(t=0)$.

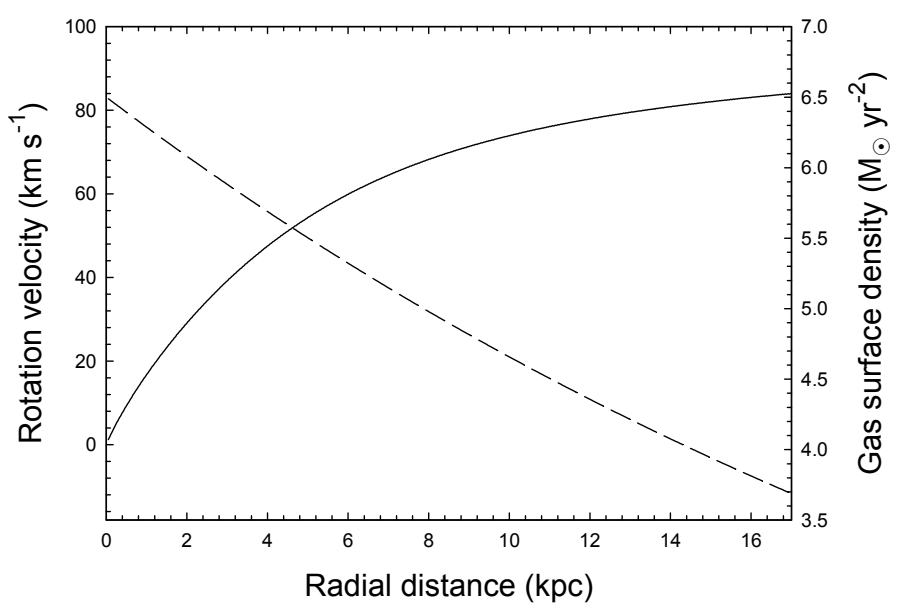

Fig. 2. The initial rotation curve (solid line) and gas surface density distribution (dashed line).

gas disk in the inner $15 \mathrm{kpc}$ is $M_{\mathrm{g}}=3.3 \times 10^{9} M_{\odot}$. Hence, the gas disk contains only a small fraction $(\sim 15 \%)$ of the total mass in the computational domain and, to a first approximation, we can neglect its self-gravity.

The initial rotation curve of the gas disk is calculated by solving the steady-state momentum equation for the radial component of the gas velocity

$\frac{u_{\phi}^{2}}{r}=\frac{R T}{\Sigma_{\mathrm{g}} \mu} \frac{\partial \Sigma_{\mathrm{g}}}{\partial r}+\frac{\partial \Phi_{\mathrm{st}}}{\partial r}+\frac{\partial \Phi_{\mathrm{h}}}{\partial r}$

where $\mu=1.2$ is the mean molecular weight for a gas of atomic hydrogen ( $76 \%$ by mass) and atomic helium ( $24 \%$ by mass) and $R$ is the universal gas constant. The initial radial gas density profile and rotation curve are shown in Fig. 2 by the dashed and solid lines, respectively. It is evident that our model LSB galaxy is characterized by a rising rotation curve with a maximum rotation velocity of approximately $80 \mathrm{~km} \mathrm{~s}^{-1}$, in agreement with the measured shapes of the rotation curve in UGC 5750 and other LSB galaxies (Kuzio de Naray et al. 2006). The main properties of our model galaxy are summarized in Table 1. 
Table 1. Main structural properties of our model galaxy.

\begin{tabular}{clcc}
\hline \hline & Mass & Scale length & Central density \\
\hline Stellar disk & $2.6 \times 10^{9}$ & 4 & 30 \\
gas disk & $3.3 \times 10^{9}$ & 30 & 6.5 \\
halo & $2.0 \times 10^{10}$ & 5.7 & $6.0 \times 10^{-3}$ \\
\hline
\end{tabular}

All masses are in $M_{\odot}$, scale lengths in kpc, and densities in $M_{\odot} \mathrm{pc}^{-2}$ (gas and stellar disks) and $M_{\odot} \mathrm{pc}^{-3}$ (halo). All masses are calculated inside $15 \mathrm{kpc}$ radius.

\subsection{Basic equations}

We use the thin-disk approximation to compute the long-term evolution of our model LSB galaxy. It is only the gas disk that is dynamically active, while both the stellar disk and dark matter halo serve as a source of external gravitational potential. In the thin-disk approximation, the vertical motion in the gas disk is neglected and the basic equations of hydrodynamics are integrated in the $z$-direction from $z=-Z(r, \phi, t)$ to $z=+Z(r, \phi, t)$ to yield the following equations in polar coordinates $(r, \phi)$

$$
\begin{aligned}
\frac{\partial \Sigma_{\mathrm{g}}}{\partial t}+\boldsymbol{\nabla}_{\mathrm{p}} \cdot\left(\boldsymbol{v}_{\mathrm{p}} \Sigma_{\mathrm{g}}\right)= & -\beta_{\mathrm{RM}} \Sigma_{\mathrm{SFR}}, \\
\frac{\partial \boldsymbol{s}_{\mathrm{p}}}{\partial t}+\boldsymbol{\nabla}_{\mathrm{p}} \cdot\left(\boldsymbol{s}_{\mathrm{p}} \cdot \boldsymbol{v}_{\mathrm{p}}\right)= & -\boldsymbol{\nabla}_{\mathrm{p}} \bar{p}-\Sigma_{\mathrm{g}} \boldsymbol{\nabla}_{\mathrm{p}} \Phi_{\mathrm{h}} \\
& -\Sigma_{\mathrm{g}} \nabla_{\mathrm{p}}\left(\Phi_{\mathrm{sp}}+\Phi_{\mathrm{st}}\right)-\boldsymbol{v}_{\mathrm{p}} \beta_{\mathrm{RM}} \Sigma_{\mathrm{SFR}}, \\
\frac{\partial \epsilon}{\partial t}+\nabla_{\mathrm{p}} \cdot\left(v_{\mathrm{p}} \epsilon\right)= & -\bar{p}(\boldsymbol{\nabla} \cdot v)+\Gamma_{\mathrm{sn}}+2 Z\left(\Gamma_{\mathrm{cr}}+\Gamma_{\mathrm{bg}}\right) \\
& -2 Z \Lambda-v_{\mathrm{p}}^{2} \beta_{\mathrm{RM}} \Sigma_{\mathrm{SFR}} .
\end{aligned}
$$

In the above equations, $\boldsymbol{v}_{\mathrm{p}}=\hat{r} v_{r}+\hat{\phi} v_{\phi}$ is the gas velocity in the disk plane, $s_{\mathrm{p}} \equiv \Sigma_{\mathrm{g}} \boldsymbol{v}_{\mathrm{p}}$ is the momentum per surface area in the disk plane, $\nabla_{\mathrm{p}}=\hat{r} \partial / \partial r+\hat{\phi} r^{-1} \partial / \partial \phi$ is the gradient along the planar coordinates of the disk, $\bar{p}$ is the vertically integrated gas pressure, $\epsilon$ is the internal energy per surface area, and $Z$ is the vertical scale height of the gas disk. The basic equations of hydrodynamics are modified to include the effect of star formation, where $\Sigma_{\mathrm{SFR}}$ is the star formation rate (SFR) per unit area and $\beta_{\mathrm{RM}}=0.42$ is the fraction of stellar mass that ends up as an inert remnant (Köppen \& Arimoto 1991). Equations (8)-(10) are closed with the equation of state of an ideal gas $\bar{p}=(\gamma-1) \epsilon$ and $\gamma=5 / 3$.

The volume cooling rate $\Lambda \equiv \Lambda\left(\mathrm{erg} \mathrm{cm}^{-3} \mathrm{~s}^{-1}\right)$ as a function of gas temperature $T$ is calculated from the cooling curves of Wada \& Norman (2001) and is linearly interpolated for metallicities between $10^{-4}$ and 1.0 of the solar. The heating of gas is provided by supernova energy release $\Gamma_{\mathrm{sn}}$, cosmic rays $\Gamma_{\mathrm{cr}}$, and a background ultraviolet radiation field $\Gamma_{\mathrm{bg}}$. The supernova energy release (per unit time and unit surface area) is defined as

$\Gamma_{\mathrm{sn}}=\frac{e_{\mathrm{sn}} \Sigma_{\mathrm{SFR}}}{m_{\mathrm{sn}}} \frac{\int_{8 M_{\odot}}^{m_{\mathrm{up}}} m \xi(m) \mathrm{d} m}{\int_{m_{\text {low }}}^{\mathrm{m}_{\mathrm{up}}} m \xi(m) \mathrm{d} m}$,

$e_{\mathrm{sn}}=10^{51}$ erg is the energy release by a single supernova, and $m_{\mathrm{sn}}=15 M_{\odot}$ is the mean mass of massive stars capable of producing supernova explosions. We use the Kroupa initial mass function (Kroupa 2001) defined as

$\xi(m)= \begin{cases}A m^{-1.3} & \text { if } m \leq 0.5 M_{\odot} \\ B m^{-2.3} & \text { if } m>0.5 M_{\odot},\end{cases}$

with the lower and upper cutoff masses $m_{\text {low }}=0.1 M_{\odot}$ and $m_{\text {up }}=$ $100 M_{\odot}$, respectively. The ratio of the normalization constants
$A / B=2$ is found using the continuity condition at $m=0.5 M_{\odot}$. We adopt the cosmic ray heating per unit time

$\Gamma_{\mathrm{cr}}=10^{-27} n_{\mathrm{g}}\left(\mathrm{erg} \mathrm{cm}^{-3} \mathrm{~s}^{-1}\right)$,

where $n_{\mathrm{g}}=\Sigma_{\mathrm{g}} /\left(2 Z \mu m_{\mathrm{H}}\right)$ is the volume number density of gas. Heating due to the background ultraviolet radiation field is supposed to balance cooling and help sustain a gas temperature $T=10^{4} \mathrm{~K}$ in the gas disk of our model galaxy unperturbed by star formation. Therefore, $\Gamma_{\mathrm{bg}}$ is determined at the beginning of numerical simulations from the relation $\Gamma_{\mathrm{bg}} n_{\mathrm{g}}=\Lambda$, where $\Lambda$ and $n_{\mathrm{g}}$ are those of the initial gas disk configuration, and is kept fixed afterwards.

The volume heating and cooling rates in Eq. (10) are assumed to be independent of the $z$-direction and are multiplied by the disk thickness (2Z) to yield the corresponding vertically integrated rates. The vertical scale height $Z \equiv Z(r, \phi, t)$ is calculated via the equation of local pressure balance in the gravitational field of the dark matter halo, stellar, and gas disks

$\rho_{\mathrm{g}} c_{s}^{2}=2 \int_{0}^{Z} \rho_{\mathrm{g}}\left(g_{z}^{\mathrm{gas}}+g_{z}^{\mathrm{st}}+g_{\mathrm{z}}^{\text {halo }}\right) \mathrm{d} z$

where $\rho_{\mathrm{g}} c_{\mathrm{s}}^{2}$ is the gas pressure in the disk midplane, $g_{z}^{\text {gas }}, g_{z}^{\text {st }}$, and $g_{z}^{\text {halo }}$ are the vertical gravitational accelerations due to selfgravity of a gas layer, stellar layer, and gravitational pull of the dark matter halo, respectively, and $c_{\mathrm{s}}^{2}=\gamma(\gamma-1) \epsilon / \Sigma_{\mathrm{g}}$ is the square of the sound speed. The details of this method are given in the Appendix. We plot $Z$ as a function of radius by the dashed line in Fig. 1 for our model gas disk at the beginning of numerical simulations. It is evident that the disk is rather thick, which is indeed expected for an intermediate-mass galaxy $\left(\sim\right.$ a few $\left.\times 10^{10} M_{\odot}\right)$ considered in the present paper. In practice, we do not let the vertical scale height drop below $100 \mathrm{pc}$, since such low values of $Z$ are not expected in LSB galaxies and may lead to overcooling of our model gas disk. It should be noticed that in our numerical simulations the energy release by supernova explosions is localized in the disk due to the adopted thin-disk approximation. In reality, some portion of the supernova energy may be lost to the intergalactic medium if the vertical scale height of a gas disk is sufficiently small and the energy release by multiple supernova explosions is sufficiently large. Given that intermediatemass LSB galaxies are characterized by thick gas disks and low rates of star formation $\left(\sim 0.1 M_{\odot} \mathrm{yr}^{-1}\right)$, we do not expect this blowout effect to considerably influence the dynamics of our model gas disk.

\subsection{Instantaneous recycling approximation}

The temporal and spatial evolution of oxygen in our model galaxy is computed adopting the so-called instantaneous recycling approximation, in which an instantaneous enrichment by freshly synthesized elements is assumed. This approximation is valid for the oxygen enrichment, because most of the oxygen is produced by short-lived, massive stars. We adopt the oxygen yields of Woosley \& Weaver (1995). We assume that oxygen is collisionally coupled to the gas, which eliminates the need to solve an additional momentum equation for oxygen. The continuity equation for the oxygen surface density $\Sigma_{\mathrm{ox}}$ modified for oxygen production via star formation reads as

$$
\frac{\partial \Sigma_{\mathrm{ox}}}{\partial t}+\nabla_{\mathrm{p}} \cdot\left(\boldsymbol{v}_{\mathrm{p}} \Sigma_{\mathrm{ox}}\right)=R_{\mathrm{OX}}-\beta_{\mathrm{RM}} \Sigma_{\mathrm{SFR}} \frac{\Sigma_{\mathrm{ox}}}{\Sigma_{\mathrm{g}}} .
$$


The oxygen production rate per unit area by supernovae is calculated as

$R_{\mathrm{OX}}=\Sigma_{\mathrm{SFR}} \frac{\int_{12 M_{\odot}}^{m_{\mathrm{up}}} p(m) \xi(m) \mathrm{d} m}{\int_{m_{\mathrm{low}}}^{m_{\mathrm{lu}}} m \xi(m) \mathrm{d} m}$,

where $p(m)$ is the mass of oxygen released by a star with mass $m$ (Woosley \& Weaver 1995). We assume that the initial oxygen abundance of our model galaxy is $[\mathrm{O} / \mathrm{H}]=\log _{10}\left(\Sigma_{\mathrm{ox}} / \Sigma_{\mathrm{g}}\right)-$ $\log _{10}\left(\Sigma_{\text {ox }} / \Sigma_{\mathrm{g}}\right)_{\odot}=-4$, where the ratio $\left(\Sigma_{\mathrm{ox}} / \Sigma_{\mathrm{g}}\right)_{\odot}$ in the Solar neighbourhood is set equal to $7.56 \times 10^{-3}$.

\subsection{Code description}

An Eulerian finite-difference code is used to solve Eqs. (8)-(10) and (15) in polar coordinates $(r, \phi)$. The basic algorithm of the code is similar to that of the commonly used ZEUS code. The operator splitting is utilized to advance in time the dependent variables in two coordinate directions. The advection is treated using the consistent transport method and the van Leer interpolation scheme. There are a few modifications to the usual ZEUS methodology, which are necessitated by the presence of star formation and cooling and heating processes in our model hydrodynamic equations. The total hydrodynamic time step is calculated as a harmonic average of the usual time step due to the CourantFriedrichs-Lewy condition and the star formation time step defined as $t_{\mathrm{SF}}=0.1 \Sigma_{\mathrm{g}} / \Sigma_{\mathrm{SFR}}$. The internal energy update due to cooling and heating in Eq. (10) is done implicitly via NewtonRaphson iterations, supplemented by a bisection algorithm for occasional zones where the Newton-Raphson method does not converge. This ensures a considerable economy in the CPU time. A typical run takes about four weeks on four $2.2 \mathrm{GHz}$ Opteron processors. The resolution is $500 \times 500$ grid zones, which are equidistantly spaced in both coordinate directions. The radial size of the grid cell is $34 \mathrm{pc}$ and the radial extent of the computational area is $17 \mathrm{kpc}$. However, star formation is confined to only the inner $15 \mathrm{kpc}$. The code performs well on the angular momentum conservation problem (see e.g. Vorobyov \& Basu 2006). This test problem is essential for the adequate modelling of rotating systems with radial mass transport.

\subsection{Sporadic star formation}

Low gas surface densities (de Blok et al. 1996; Pickering 1999), which are usually below the critical value determined by the Toomre criterion, and low total star formation rates (van den Hoek et al. 2000) argue against large-scale star formation as a viable scenario for LSB galaxies. Despite the low gas surface densities, there is evidence for a noticeable young stellar population as indicated by the blue colors of most LSBs (de Blok et al. 1995). According to de Blok et al. (1995) and Gerretsen \& de Blok (1999), a sporadic star formation scenario (i.e. small surges in the SFR, either superimposed on a very low constant SFR or not) can best explain the observed blue colors and evidence for young stars.

There is little known about physical processes that control sporadic star formation in LSB galaxies. The recent uncalibrated $\mathrm{H} \alpha$ imaging of a sample of LSB galaxies by Auld et al. (2006) and the observational data presented here (see Sect. 4) suggest that star formation is highly clustered. Current star formation is localized to a handful of compact regions. There is little or no diffuse $\mathrm{H} \alpha$ emission coming from the rest of the galactic disk.
Motivated by this observational evidence, we adopt the sporadic scenario for star formation in our model LSB galaxy.

We use a simple model of sporadic star formation in which the individual star formation sites (SFSs) are distributed randomly throughout the galactic disk and the star formation rate per unit area in each SFS is determined by a Schmidt law

$\frac{\Sigma_{\mathrm{SFR}}}{M_{\odot} \mathrm{yr}^{-1} \mathrm{kpc}^{-2}}=\alpha_{\mathrm{SF}}\left(\frac{\Sigma_{\mathrm{g}}}{M_{\odot} \mathrm{pc}^{-2}}\right)^{1.5}$ if $T<T_{\mathrm{cr}}$,

where $\alpha_{\mathrm{SF}}=6 \times 10^{-4}$ is a proportionality constant and $T_{\mathrm{cr}}=$ $2 \times 10^{4} \mathrm{~K}$. If the gas temperature in a randomly chosen SFS exceeds the critical value, the SFS is rejected. The adopted value of $T_{\text {cr }}=2 \times 10^{4} \mathrm{~K}$ differs from typical temperatures of starforming molecular clouds: $T \lesssim 30-50 \mathrm{~K}$. They are however more appropriate for identification of star-forming regions on $\sim 34$ pc scales that are resolved in the present numerical simulations. A Schmidt law with index $n \sim 1.5$ would be expected for self-gravitating disks, if the SFR is equal to the ratio of the local gas volume density $(\rho)$ to the free-fall time $\left(\propto \rho^{-0.5}\right)$, all multiplied by some star formation efficiency $\epsilon$. The efficiency $\epsilon$ is a measure of the fraction of the gas mass converted into stars before the clouds are disrupted. It can be shown that $\epsilon$ is related to $\alpha_{\mathrm{SF}}$ as (Vorobyov 2003)

$\alpha_{\mathrm{SF}}=0.12 \epsilon\left(\frac{Z}{\mathrm{pc}}\right)^{-0.5}$.

This means that the adopted star formation efficiency in our model ranges between 0.045 and 0.15 for possible values of the vertical scale height $Z=100-1500 \mathrm{pc}$.

The number of star formation sites at any given time can be roughly determined using the uncalibrated $\mathrm{H} \alpha$ images of LSB galaxies by Auld et al. (2006). These images highlight only a handful of small regions that have recently formed stars. There is little diffuse $\mathrm{H} \alpha$ emission, contrary to the emission in $K-, R$-, and $B$-bands which (as a rule) show a noticeable diffuse component. The number of HII regions within an individual LSB galaxy identified by van den Hoek et al. (2000) ranges between 4 and 26, with a median number of HII regions equal to 11 . Therefore, the number of SFS, $N_{\mathrm{SFS}}$, in our numerical simulations is set to approximately 15 . If we further assume that both $N_{\mathrm{SFS}}$ and the typical area occupied by an individual star formation site $S_{\text {SFS }}$ do not change significantly with time, this would yield a near-constant mean SFR over the lifetime of our model galaxy. However, there is evidence that star formation in blue LSB galaxies does not proceed at a near-constant rate. Our own numerical simulations and modeling by Zackrisson et al. (2005) indicate that the SFR should be declining with time to reproduce the observed $\mathrm{H} \alpha$ equivalent widths in LSB galaxies. Therefore, we assume that the typical area occupied by a SFS is a decreasing function of time of the form $S_{\mathrm{SFS}}=S_{\mathrm{SFS} 0} \exp \left(-t / \tau_{\mathrm{SFR}}\right)$, where $S_{\mathrm{SFS} 0}=450 \times 450 \mathrm{pc}$ is the area at $t=0$ and $\tau_{\mathrm{SFR}}=$ $1.3 \mathrm{Gyr}$ is a characteristic time of exponential decay in the star formation rate.

The duration of star formation in an individual star formation site $\left(\tau_{\mathrm{SFS}}\right)$ is poorly known. We use the theoretical and empirical knowledge gained by analyzing similar processes in the Solar neighbourhood. Observations of the current star formation activity in nearby molecular cloud complexes indicate that the ages of T Tauri stars usually fall in the 1-10 Myr range (Palla \& Stahler 2002). Clusters that are $10 \mathrm{Myr}$ old often have little associated gas remaining, implying that the process shuts off by then. However, the volume gas density in disks of LSB galaxies 
is expected to be lower (on average) than that of the Solar neighbourhood, which may prolong the process of gas conversion into stars. It should also be noted that individual star formation sites in LSB galaxies have characteristic sizes that are much larger than those of an individual giant molecular cloud. Otherwise, we would have to assume unrealistically high star formation efficiency in LSB galaxies to retrieve the observed star formation rates of the order of $0.1 M_{\odot} \mathrm{yr}^{-1}$. This implies a presence of $l o$ cal triggering mechanisms within an individual SFS that should also act to prolong the star formation activity. From the theoretical background, one can argue that the duration of star formation should be limited by feedback from newly born massive stars and supernova explosions. The lifetime of the least massive star capable of producing a supernova is approximately $40 \mathrm{Myr}$. Hence, the actual lifetime of an individual SFS should lie in between $10 \mathrm{Myr}$ and $40 \mathrm{Myr}$. Using spectro-photometric evolution models, van den Hoek et al. (2000) have also found that $\tau_{\mathrm{SFS}}=10-50$ Myr reproduce well the observed $(R-I)$ colors and $I$-band luminosities of LSB galaxies. In our numerical simulations we assume that $\tau_{\mathrm{SFS}}=20 \mathrm{Myr}$. Thus, each twenty million years we initialize a new set of SFSs $\left(N_{\mathrm{SFS}} \approx 15\right)$ and randomly distribute them in the inner $15 \mathrm{kpc}$ of the galactic disk. Each SFS is assigned a velocity equal to that of the local gas velocity upon its creation and is evolved collisionlessly in the total gravitational potential of the stellar disk and the dark matter halo using the fifth-order Runge-Kutta Cash-Karp integration scheme. The old SFSs are rendered inactive and their further evolution is not computed.

\section{Observations}

There is only a small number of (nearby) LSB galaxies with published $\mathrm{H} \alpha$ images, which would allow the study of the distribution and properties of their star-formation regions. $\mathrm{H} \alpha$ images of about 18 LSB galaxies are available in McGaugh \& Bothun (1994); McGaugh et al. (1995b), while de Blok \& van der Hulst (1998) only published the spectra and some broad band images. A new sample of 6 LSB galaxies was presented in Auld et al. (2006).

From the image data set (McGaugh, priv. comm.) we selected 4 LSB spiral galaxies based on their $B$ band morphology. We selected the galaxies to be close to face-on and showing different disk surface brightness and spiral morphology. This resulted in UGC 9024, UGC 12845, UGC 5675, and UGC 6151. A different version of the $\mathrm{H} \alpha$ images of UGC 9024, UGC 5675, and UGC 6151 was previously published in McGaugh et al. (1995b).

For in Fig. 3 we improved the cosmic rays rejection and correction of detection blemishes of the already flat-fielded images. The generation of the continuum corrected $\mathrm{H} \alpha$ images followed closely the schema described e.g. in Bomans (1997). We realigned the $\mathrm{H} \alpha$ and continuum images carefully using the foreground stars visible in both images to sub-pixel accuracy and subtracted the scaled continuum image. We tested different scaling factors and defined an optimal subtraction based on the disappearance of the stellar disk of the LSB galaxy. These factors also gave the smallest foreground star residuals and is consistent with the scaling factor calculated by measuring the flux of several foreground stars in both images.

The distribution of the HII regions in all 4 galaxies in Fig. 3 is consistent with the predicted pattern of sporadic star formation. The $\mathrm{H} \alpha$ images of 6 LSB galaxies recently published by
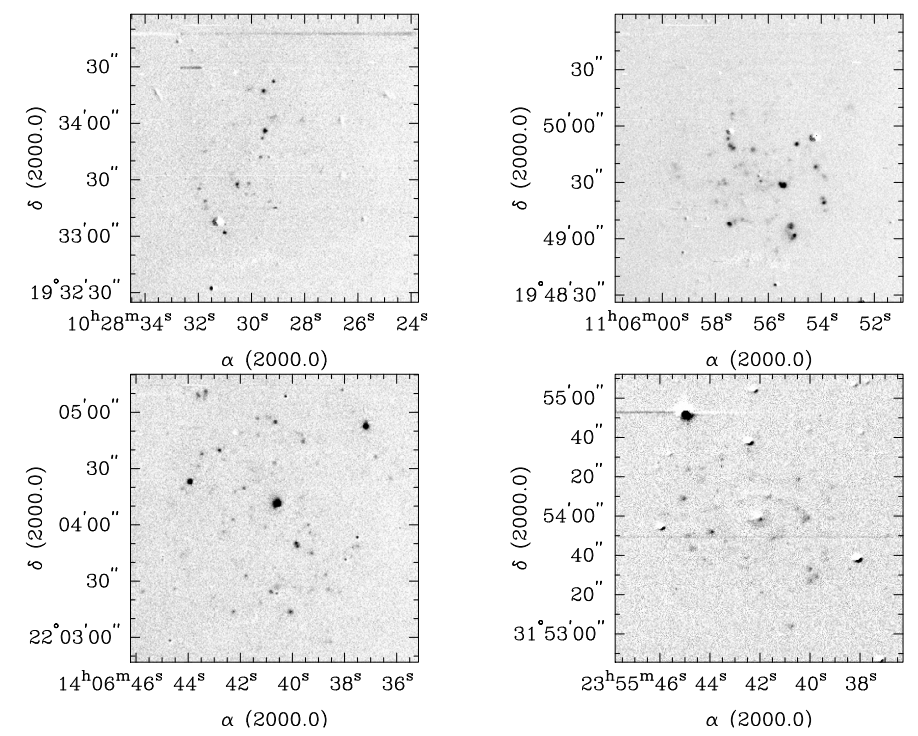

Fig. 3. Reprocessed and continuum subtracted images of the LSB galaxies UGC 9024 (upper left), UGC 12845 (upper right), UGC 5675 (lower left), and UGC 6151 (lower right).

Auld et al. (2006) show the same behavior, as do the other galaxies published in McGaugh et al. (1995b).

\section{Numerical results}

\subsection{Gas density and temperature distribution}

The model galaxy described in Sect. 3 will be referred hereafter as model 1. We will also introduce other models that have identical structural properties such as those summarized in Table 1 but different IMFs and star formation rates. We start our numerical simulations by setting the equilibrium gas disk and slowly introducing the non-axisymmetric gravitational potential of the stellar spiral arms. Specifically, $\Phi_{\mathrm{sp}}$ is multiplied by a function $\xi(t)$, which has a value of 0 at $t=0$ and linearly grows to its maximum value of 1.0 at $t \geq 200 \mathrm{Myr}$. It takes another hundred million years for the gas disk to adjust to the spiral gravitational potential and develop a spiral structure. Figure 4 shows the gas surface density distribution (in log units) in the 1 Gyr old (upper left), 2 Gyr old (upper right), 5 Gyr old, (lower left), and 12 Gyr old (lower right) disks. It is seen that the gas disk develops a strong phase separation with age. While the initial gas disk has surface densities between $3.5 M_{\odot} \mathrm{pc}^{-2}$ and $6.5 M_{\odot} \mathrm{pc}^{-2}$, the 1-Gyr-old disk features some dense clumps with surface densities in excess of $100 M_{\odot} \mathrm{pc}^{-2}$ and temperatures of $100-200 \mathrm{~K}$. The number of such clumps increases with time and they are stretched into extended filaments and arcs by galactic differential rotation. Observational detection of such features could be a powerful test on the sporadic nature of star formation in LSB galaxies. The gas response to the underlying spiral stellar density wave is rather weak in the early disk evolution and can hardly be noticed in the 5 and 13 Gyr old disks. This implies that mixing due to spiral stellar density waves is inefficient in the old disk.

The phase separation of the gas disk can be illustrated by calculating the gas mass per unit temperature band at different evolutionary times. Figure 5 shows the gas mass per Kelvin as a function of temperature at $t=2 \mathrm{Gyr}$ (solid line), $t=5 \mathrm{Gyr}$ (dashed line) and $t=13 \mathrm{Gyr}$ (dash-dotted line). There are 


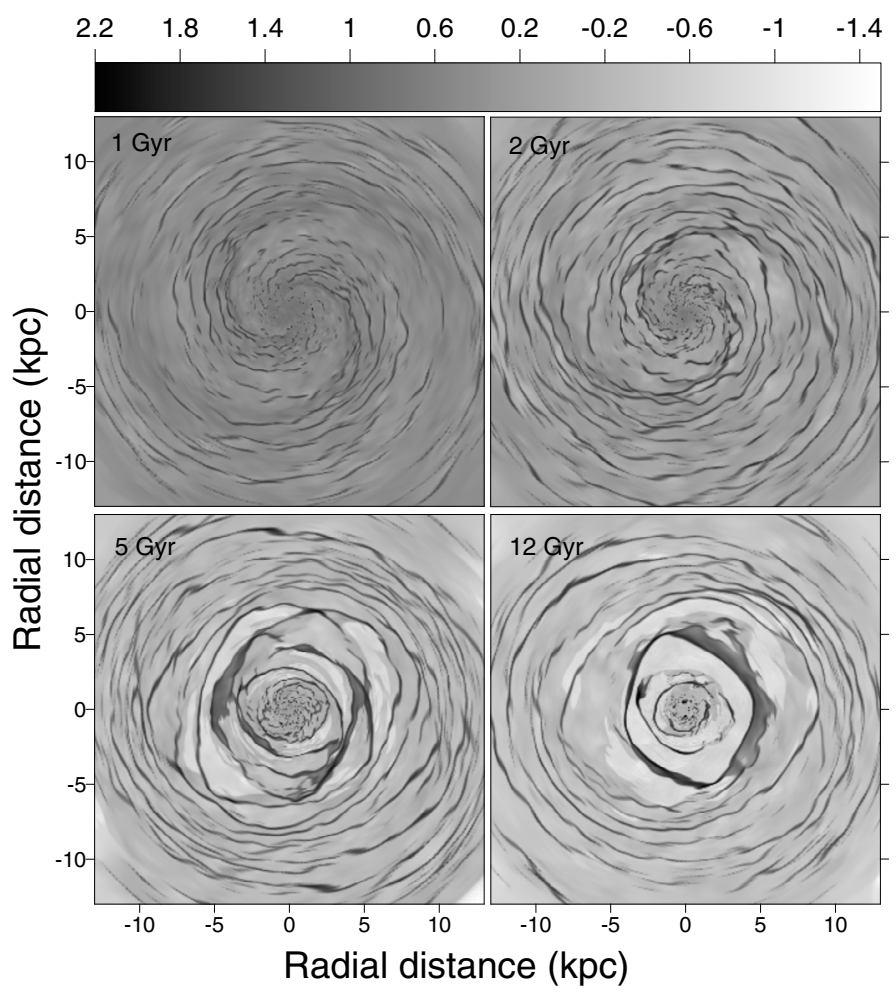

Fig. 4. Gas surface density distribution in the 1-Gyr-old (upper left), 2-Gyr-old (upper right), 5-Gyr-old (lower left), and 12-Gyr-old (lower right) disks. Dark regions are the densest and coldest. The scale bar is $M_{\odot} \mathrm{pc}^{-2}$ (log units).

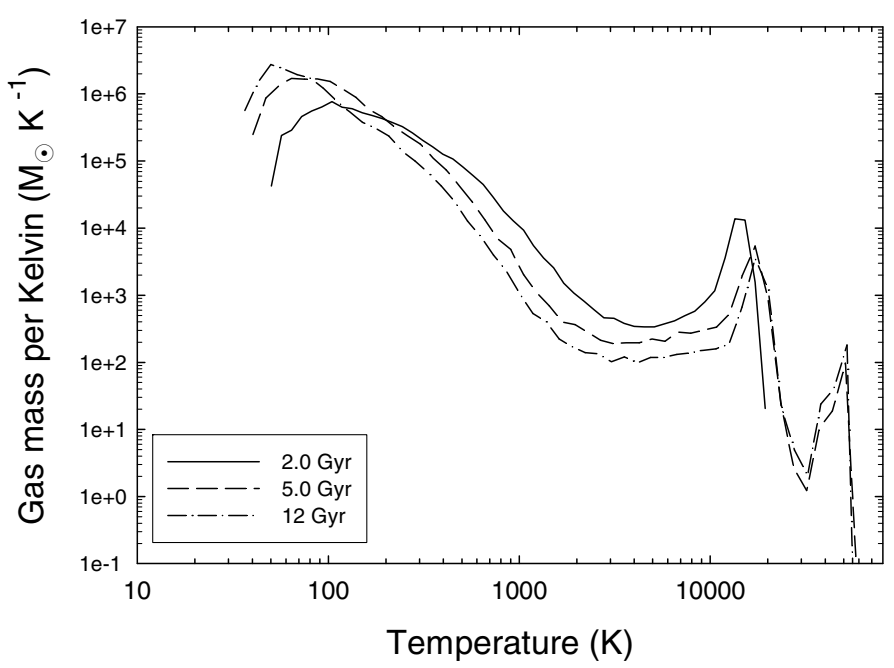

Fig. 5. Gas mass per Kelvin versus temperature in disks of distinct age as indicated in the legend. The two peaks manifest the phase separation. Note that there is little gas with a temperature below $40 \mathrm{~K}$.

two prominent peaks that manifest the separation of the gas into two phases - a cold gas phase with a peak temperature of $T=50-100 \mathrm{~K}$ and a warm gas phase with a peak temperature of $T=(1.0-1.5) \times 10^{4} \mathrm{~K}$. The positions of the peaks drift apart as the gas disk evolves, indicating that the cold phase becomes colder and the warm phase becomes warmer with time. However, the gas temperature never drops below $35 \mathrm{~K}$. It is also evident from the amplitudes of the peaks that the cold phase accumulates gas mass with time, which is explained by increased cooling due to ongoing heavy element production. A similar

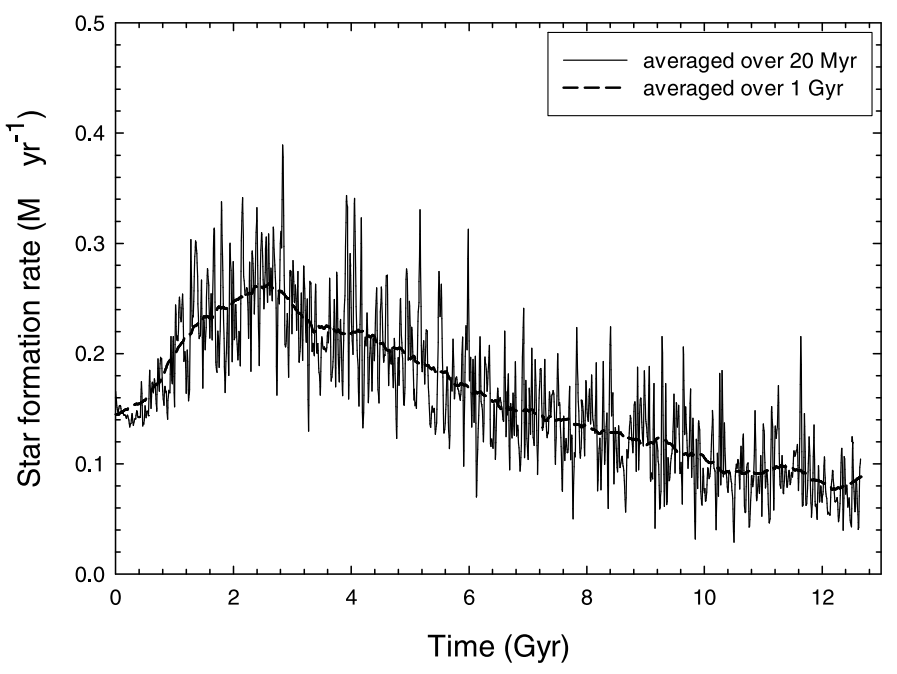

Fig. 6. Star formation rate versus time in model 1. The solid and thick dashed lines show the SFR averaged over $20 \mathrm{Myr}$ and $1.0 \mathrm{Gyr}$, respectively. Note large amplitude fluctuations in the $20 \mathrm{Myr}$ averaged SFRs around the $1 \mathrm{Gyr}$ averaged values.

phase separation was also obtained in numerical simulations of LSBs by Gerretsen \& de Blok (1999). The fact that there is little gas with temperature below $35 \mathrm{~K}$ implies that most of the cold phase may be in the form of atomic rather than molecular hydrogen, but more accurate numerical simulations with a chemical reaction network and higher numerical resolution are needed to prove this hypothesis.

Figure 5 indicates that model 1 has no hot gas phase with temperatures of order $10^{6} \mathrm{~K}$, which is a consequence of a relatively low SFR and moderate numerical resolution. The energy injection by star formation of such a low rate is not enough to convert all gas in a computational cell $(\sim 34 \times 34 \mathrm{pc})$ to the hot phase. This is because we solve the equations of hydrodynamics for a single fluid. As a consequence, the temperature of all gas in a computational cell is characterized by a specific value and there is no gas phase separation within an individual cell. This, however, does not mean that the hot gas is not present at all in LSB galaxies, we simply may need a much higher numerical resolution to resolve it. We do start seeing the hot phase if we increase the SFR in our model by a factor of several. We note that Gerretsen \& de Blok (1999) have also found little hot gas in their numerical simulations.

Figure 6 shows the SFR as a function of time in model 1. In particular, the solid line presents the SFR averaged over the past $20 \mathrm{Myr}$; these values are later used to construct $\mathrm{H} \alpha$ equivalent widths. In addition, the thick dashed line plots the SFR averaged over a 1 Gyr interval centered on the current time (running average method). It is seen that the $20 \mathrm{Myr}$ averaged SFRs have considerable fluctuations around the $1 \mathrm{Gyr}$ averaged values. These fluctuations have a characteristic time scale of a few hundred Myr and amplitudes that may exceed $0.1 M_{\odot} \mathrm{yr}^{-1}$. The existence of such bursts of star formation is an essential ingredient for successful modelling of blue colors of at least some LSB galaxies (e.g. van den Hoek et al. 2000). Similar fluctuations in the SFR were also observed in numerical simulations by Gerretsen \& de Blok (1999). The 1 Gyr averaged SFR quickly grows to a maximum value $0.26 M_{\odot} \mathrm{yr}^{-1}$ at $t=2.6 \mathrm{Gyr}$ and subsequently declines to $0.08 M_{\odot} \mathrm{yr}^{-1}$ at $t=13.0 \mathrm{Gyr}$. Typical star formation rates in LSB galaxies range from 0.02 to $0.2 M_{\odot} \mathrm{yr}^{-1}$, with a median SFR of $0.08 M_{\odot} \mathrm{yr}^{-1}$ (van den Hoek et al. 2000). Model 1 


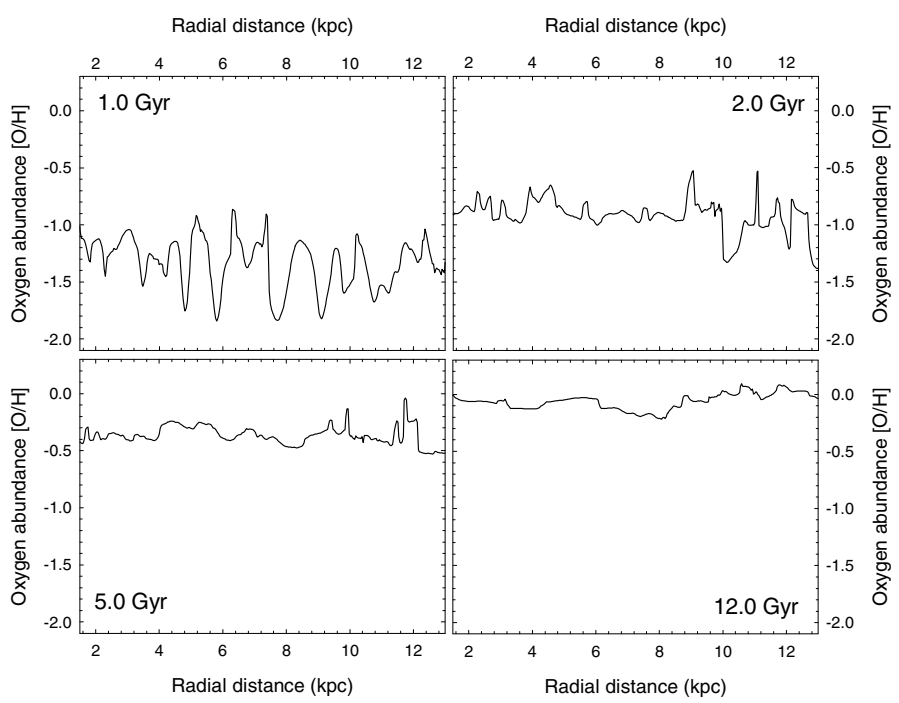

Fig. 7. Radial profiles of $[\mathrm{O} / \mathrm{H}]$ obtained by making a narrow cut through the galactic disk at an azimuthal angle $\phi=0^{\circ}$. The age of the disk is indicated in each frame.

yields SFRs that are consistent with the inferred present-day rates.

\subsection{Radial variations in the oxygen abundance}

We calculate the oxygen abundance $[\mathrm{O} / \mathrm{H}]=\log _{10}\left(\Sigma_{\mathrm{ox}} / \Sigma_{\mathrm{g}}\right)-$ $\log _{10}\left(7.56 \times 10^{-3}\right)$ in our disk from the model's known surface densities of gas $\left(\Sigma_{\mathrm{g}}\right)$ and oxygen $\left(\Sigma_{\mathrm{ox}}\right)$. We find that the spatial distribution of oxygen in the 1 Gyr old gas disk has a pronounced patchy appearance - local regions with enhanced oxygen abundance adjoin local regions with low oxygen abundance. The local contrast in $[\mathrm{O} / \mathrm{H}]$ may be as large as 1.0 dex or even more. As the galaxy evolves, the spatial distribution of oxygen becomes noticeably smoother. The smearing is caused by stirring due to differential rotation, radial migration of gas triggered by the non-axisymmetric gravitational potential of stellar spiral arms, and energy release of supernova explosions.

To better illustrate the spatial variations in the oxygen distribution in model 1, we show in Fig. 7 the radial profiles of the oxygen abundance obtained by making a narrow $(\sim 50 \mathrm{pc})$ radial cut through the galactic disk at $\phi=0^{\circ}$. The numbers in each frame indicate the age of our model galaxy. The most prominent feature in Fig. 7 is a sharp decline in the typical amplitude of the radial fluctuations in $[\mathrm{O} / \mathrm{H}]$. For instance, the radial distribution of the oxygen abundance in the 1 Gyr old disk is very irregular and shows radial fluctuations with amplitudes as large as $1.0 \mathrm{dex}$ and even more. The fluctuations have a short characteristic radial scale of the order of 1-2 kpc. The fluctuation amplitudes have noticeably decreased in the 2 Gyr old disk, which now has a maximum amplitude of the order of 0.5 dex. The 5 Gyr old disk (and older disks) feature only low-amplitude fluctuations in the oxygen abundance with amplitudes rarely exceeding 0.2 dex.

Figure 7 reveals the absence of any significant radial abundance gradients in our model disk. This appears to be typical of LSB galaxies (e.g. de Blok \& van der Hulst 1998). The lack of radial abundance gradients is most likely caused by a spatially and temporally sporadic nature of star formation, i.e., LSB galaxies must have evolved at the same rate over their entire disk. Well-known star formation threshold criteria, such as those based on the gas surface density threshold (Toomre criterion) or the rate of shear (Martin \& Kennicutt 2001; Vorobyov 2003),

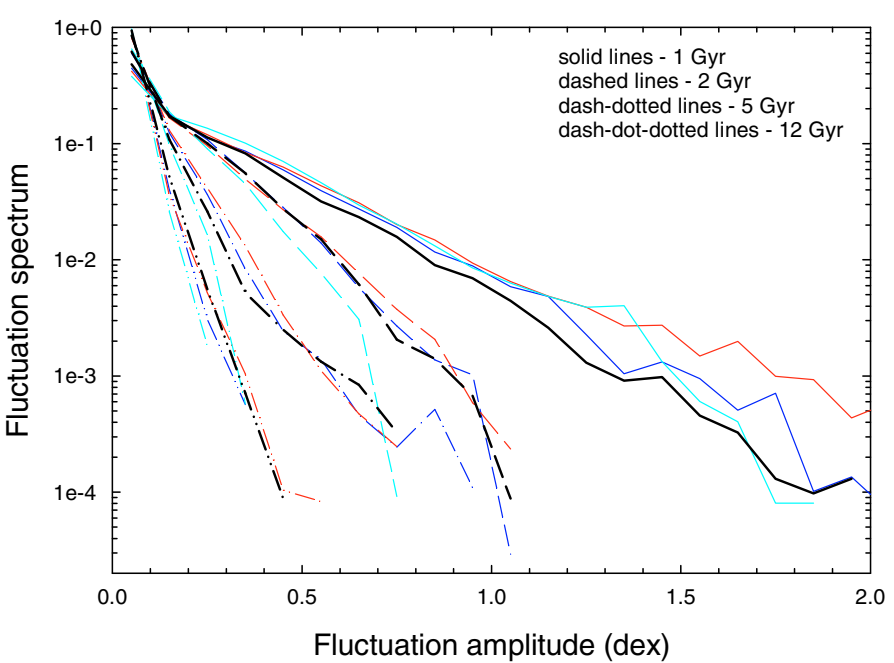

Fig. 8. Oxygen abundance fluctuation spectrum defined as the normalized number of radial oxygen abundance fluctuations versus fluctuation amplitude (in dex). Lines of distinct color correspond to different numerical models and lines of distinct type correspond to different galactic ages as indicated. See the text for more details.

would increase the likelihood of star formation in regions of higher density and lower angular velocity, which are usually the inner galactic regions. As a result, negative radial abundance gradients may develop with time. The fact that we do not see radial abundance gradients in LSB galaxies implies that star formation has no threshold barrier.

To analyze the properties of the oxygen abundance fluctuations in disks of different age, we calculate the fluctuation spectrum defined as the (normalized) number of fluctuations versus the fluctuation amplitude $A$. The latter is calculated in the following manner. We scan the disk in the radial direction along each azimuthal angle $(\phi)$ of our numerical polar grid for the adjacent minima and maxima in the oxygen abundance distribution. The absolute value of the difference between the adjacent minimum and maximum (or vice versa) gives us the radial amplitude of a fluctuation on spatial scales of our numerical resolution, $\sim 40 \mathrm{pc}$. The calculated fluctuation amplitudes are distributed in $40 \mathrm{log}$ arithmically spaced bins between 0.1 dex and 3.0 dex and then normalized by the total number of fluctuations in all bins. The resulting fluctuation spectrum has the meaning of the probability function $\mathcal{F}(A)$ - the product of the total number of fluctuations and $\mathcal{F}(A)$ yields the number of fluctuation with amplitude $A$.

Figure 8 shows the probability function $\mathcal{F}(A)$ derived for four models detailed below. Hereafter, different line types in Fig. 8 will help to distinguish between galaxies of distinct age and different line colors will correspond to different numerical models. Black lines present $\mathcal{F}(\mathcal{A})$ in model 1 for the $1 \mathrm{Gyr}$ old disk (solid line), 2 Gyr old disk (dashed line), 5 Gyr old disk (dash-dotted line), and 12 Gyr old disk (dash-dot-dotted line). It is evident that the probability functions for disks of different ages intermix for $\mathcal{F}(A) \gtrsim 0.1$ but appear to be quite distinct for $\mathcal{F}(A) \lesssim 0.05$. Can this peculiar feature of $\mathcal{F}(A)$ be used to constrain the age of LSB galaxies? The extent to which oxygen is spatially mixed in the galactic disk may depend on the model parameters such as the strength of the spiral density wave, shape of the IMF, and details of the energy release by SNe. In order to estimate the dependence of $\mathcal{F}(A)$ on these factors, we run two test models. Model T1 has no spiral density waves and model T2 is characterized by a factor of 2 lower energy input by $\mathrm{SNe}$ ("T" stands for "test" to distinguish them from regular 
Table 2. Summary of galactic models.

\begin{tabular}{lccccc}
\hline \hline Model & IMF type & Upper/lower cutoffs & $\tau_{\mathrm{SFR}}$ & $\alpha_{\mathrm{SF}}$ & $\min / \max S F R$ \\
\hline 1 & Kroupa & $0.1-100 M_{\odot}$ & 13.0 & $6 \times 10^{-4}$ & $0.075 / 0.26$ \\
$1 \mathrm{~L}$ & Kroupa & $0.1-100 M_{\odot}$ & 13.0 & $4 \times 10^{-4}$ & $0.085 / 0.17$ \\
2 & Salpeter & $0.1-100 M_{\odot}$ & 18.5 & $6 \times 10^{-4}$ & $0.045 / 0.20$ \\
2L & Salpeter & $0.1-100 M_{\odot}$ & 18.5 & $4 \times 10^{-4}$ & $0.050 / 0.14$ \\
\hline
\end{tabular}

Star formation rate is in $M_{\odot} \mathrm{yr}^{-1}$ and characteristic time of SFR decay $\tau_{\mathrm{SFR}}$ is in Gyr.

models). Model T2 attempts to mimic the case when part of the $\mathrm{SN}$ energy is lost to the extragalactic medium via SN bubbles. It is also important to see the effect that a different IMF may have on the probability function. Therefore, we introduced model 2, which is characterized by the Salpeter IMF with the upper and lower cut-off masses of $0.1 M_{\odot}$ and $100 M_{\odot}$. Model 2 has the same structural parameters as model 1 except for $\tau_{\text {SFR }}$ which is set to 18.5 Gyr. The parameters of models 1 and 2 are detailed in Table 2. The probability functions corresponding to model T1, model T2, and model 2 are shown in the top panel of Fig. 8 by the red, blue, and cyan lines, respectively. The meaning of the line types (i.e., solid, dashed, etc.) is indicated in the figure.

A noticeable scatter in the probability functions of distinct age is seen among different models. In particular, the probability functions of galaxies of 5-12 Gyr age intermingle and thus cannot be used to make age predictions. However, it is still possible to differentiate between (1-2) Gyr old and (5-12) Gyr old galaxies based on the slope of $\mathcal{F}(A)$. Young galaxies of 1-2 Gyr age have fluctuation spectra with a much shallower slope than (5-13) Gyr old galaxies, meaning that the former feature radial oxygen abundance fluctuations of much larger amplitude than the latter. A similar behaviour of $\mathcal{F}(A)$ is found when we decrease the spatial resolution of the fluctuation amplitude measurements from $40 \mathrm{pc}$ to several hundred parsecs (more appropriate for modern observational techniques). We conclude that measurements of the radial oxygen abundance fluctuations may be used only to set order-of-magnitude constraints on the age of blue LSB galaxies.

We are aware of only one successful measurement of the radial abundance gradients in three LSB galaxies done by de Blok \& van der Hulst (1998). Unfortunately, the number of resolved HII regions and oxygen abundance measurements in each galaxy is too low $(<10)$ to make a meaningful comparison with our predictions. With such a small sample, the probability function would be confined in the $0.1<\mathcal{F}(A)<1.0$ range, which is not helpful for making age predictions due to degeneracy of $\mathcal{F}(A)$ in this range.

\section{Constraining the age of blue LSB galaxies}

In this section, we investigate if the time evolution of the mean oxygen abundance, complemented by $\mathrm{H} \alpha$ emission equivalent widths and optical colors, can be used to better constrain the age of blue LSB galaxies. We consider several new models (in addition to our models 1 and 2) that have different rates of star formation.

\subsection{Time evolution of the oxygen abundance}

There have been several efforts in the past to measure the oxygen abundances in HII regions of blue LSB galaxies. For instance, oxygen abundances in a sample of 18 galaxies in Kuzio de Narray et al. (2004) have a median value $\langle[\mathrm{O} / \mathrm{H}]\rangle=-0.95$. Their

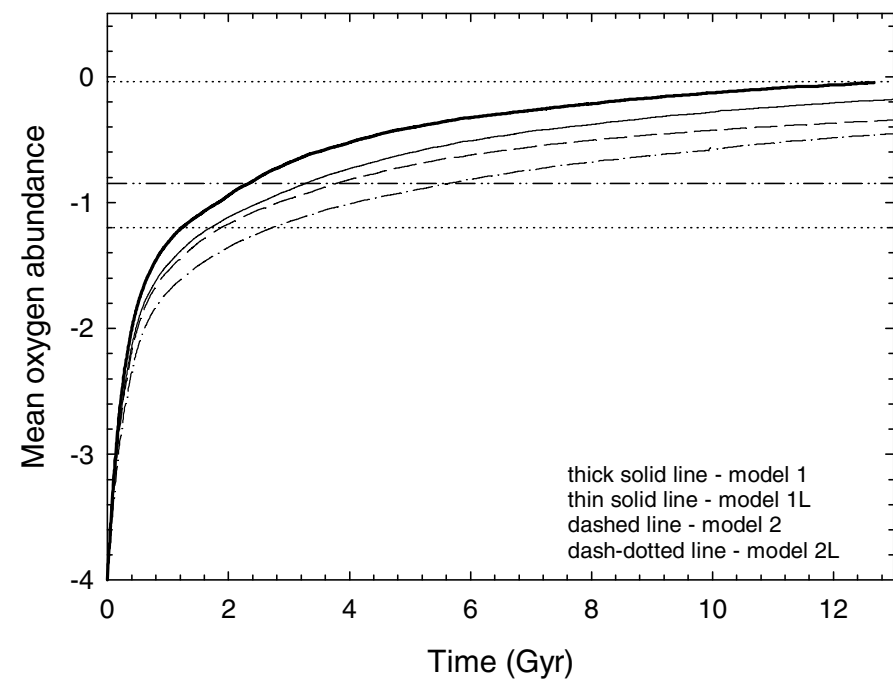

Fig. 9. Mean oxygen abundance as a function of galactic age. Each line type corresponds to a particular numerical model as indicated in the figure. The horizontal dotted lines delineate the range of oxygen abundances found in blue LSB galaxies (de Blok \& van der Hulst 1998; Kuzio de Narray et al. 2004) and the horizontal dashed-dot-dotted line shows the mean oxygen abundance derived from a large sample of LSB galaxies. See the text and Table 2 for more details.

smallest and largest measured abundances (using the equivalent width method) are $[\mathrm{O} / \mathrm{H}]=-1.2(\mathrm{~F} 611-1)$ and $[\mathrm{O} / \mathrm{H}]=-0.04$ (UGC 5709). de Blok \& van der Hulst (1998) have observed 64 HII regions in 12 LSB galaxies and derived oxygen abundances that peak around $[\mathrm{O} / \mathrm{H}]=-0.80$. The lowest abundance in their sample is $[\mathrm{O} / \mathrm{H}]=-1.1$. These data demonstrate that the present day mean oxygen abundance in LSB galaxies is about $\langle[\mathrm{O} / \mathrm{H}]\rangle_{\mathrm{LSB}}=-0.85 \mathrm{dex}$, albeit with a wide scatter. We plot the minimum and maximum oxygen abundances found in blue LSB galaxies by horizontal dotted lines in Fig. 9, while the mean oxygen abundance for LSB galaxies is shown by the vertical dashdot-dotted line.

The amount of produced oxygen and the oxygen abundance are expected to depend on the adopted rate of star formation and on the details of the initial mass function. Most of oxygen is produced by intermediate to massive stars and both the slope and the upper mass cutoff are those parameters of the IMF that may influence the rate of oxygen production. In this paper, we study IMFs of different slope, leaving cut-off IMFs for a subsequent study.

The mean oxygen abundances $\langle[\mathrm{O} / \mathrm{H}]\rangle$ obtained in model 1 are presented in Fig. 9 by the thick solid line. The mean values are calculated by summing up local oxygen abundances in each computational cell between $1 \mathrm{kpc}$ and $14 \mathrm{kpc}$ and then averaging over the corresponding number of cells. The dashed line shows $\langle[\mathrm{O} / \mathrm{H}]\rangle$ as a function of galactic age in model 2 . In addition, we have introduced two more models that have structural 
parameters identical to those of models 1 and 2 (see Table 1) but are characterized by lower SFRs. In particular, models $1 \mathrm{~L}$ and 2L (thin solid line/dash-dotted line) feature on average a factor of 1.4 lower rates of star formation than models 1 and 2. The parameters of models $1 \mathrm{~L}$ and $2 \mathrm{~L}$, as well as the minimum and maximum SFRs, are listed in Table 2. It is evident that models with the Salpeter IMF yield lower $\langle[\mathrm{O} / \mathrm{H}]\rangle$ than the corresponding models with the Kroupa IMF. With the lower and upper mass limits being identical, the Sapleter IMF may be regarded bottomheavy in comparison to that of Kroupa. A bottom-heavy IMF has a lower relative number of massive stars and this fact accounts for the lower rate of oxygen production in models with the Salpeter IMF. Furthermore, models with lower SFRs yield lower mean oxygen abundances.

There are several interesting conclusions that can be drawn by analyzing Fig. 9. First, our models can reproduce the entire range of observed oxygen abundances found in LSB galaxies (delimited by horizontal dotted lines). Second, for a given value of $\langle[\mathrm{O} / \mathrm{H}]\rangle$, the predicted ages show a large scatter (that increases along the sequence of increasing oxygen abundances), making mean oxygen abundances alone a poor age indicator, especially for metal-rich LSBs. Finally, the minimum oxygen abundance found among LSB galaxies (bottom dotted line in Fig. 9) can be attained after just 1-3.5 Gyr of evolution, suggesting a possibility of rather young age for LSB galaxies. We will return to this issue in some more detail in the following section.

\subsection{Ho equivalent widths and B-V integrated colors}

In this section we use population synthesis modelling to calculate the integrated $B-V$ colors and $\mathrm{H} \alpha$ equivalent widths $(E W(\mathrm{H} \alpha))$ using the model's known SFRs and mean oxygen abundances. The interested reader can find details of the population synthesis model and essential tests in the Appendix. There is observational evidence that LSB galaxies are characterized by $B-V$ colors and $E W(\mathrm{H} \alpha)$ that lie in certain limits. For instance, the observed $B-V$ colors of blue LSB galaxies (which we attempt to model in this paper) appear to lie in the $0.3-0.7$ range (de Blok et al. 1995). According to Zackrisson et al. (2005), $E W(\mathrm{H} \alpha)$ of a sample of blue LSB galaxies seem to be confined within rather narrow bounds, $E W(\mathrm{H} \alpha)=10-60 \AA$. Hence, population synthesis modelling may be regarded as a consistency test for our numerical models, which should yield $E W(\mathrm{H} \alpha)$ and $B-V$ colors in acceptable agreement with observations.

We present the results of population synthesis modelling only for models with different IMFs; models with different SFRs show a similar behaviour. Figure 10 shows model $E W(\mathrm{H} \alpha)$ (left column) and $B-V$ colors (right column) as a function of galactic age for model 1 (top) and model 2 (bottom). The equivalent widths are derived using SFRs averaged over the past $20 \mathrm{Myr}$. For convenience, the thick dashed lines show $E W(\mathrm{H} \alpha)$ averaged over 1 Gyr. The horizontal dotted lines in the left panels show a typical range for $E W(\mathrm{H} \alpha)$ in blue LSB galaxies (Zackrisson et al. 2005), while the horizontal dotted lines in the right panels outline typical $B-V$ colors of blue LSB galaxies (de Blok et al. 1995). The arrow shows a (possible) reddening due to dust extinction for a fiducial value of the color excess $E(B-V)=0.1$ (McGaugh 1994).

It is evident that model $E W(\mathrm{H} \alpha)$ show considerable fluctuations of the order of 15-20 $\AA$ around mean values, which reflect the corresponding fluctuations in the SFR. Neither model 1 nor model 2 can account for the observed equivalent widths in the early evolution, yielding too large $E W(\mathrm{H} \alpha)$ during the first

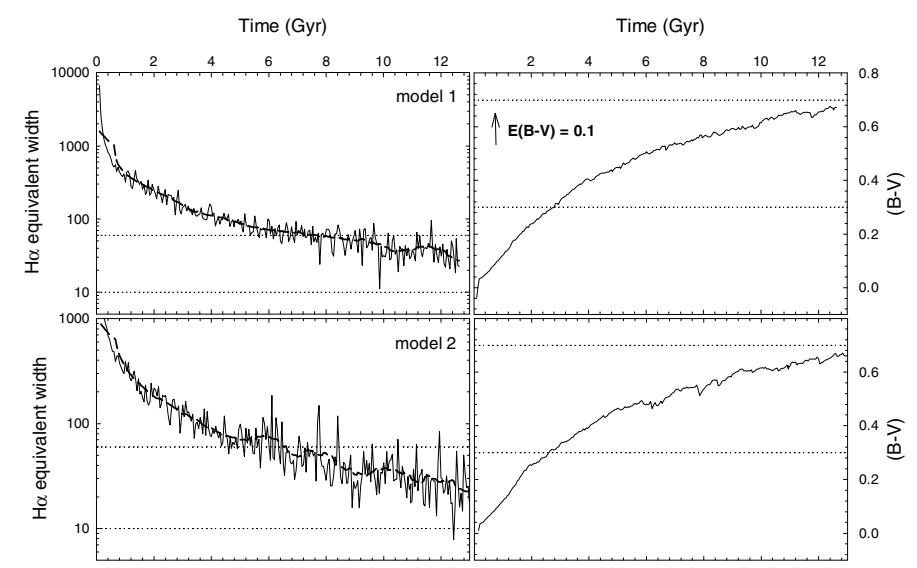

Fig. 10. $\mathrm{H} \alpha$ equivalent widths (left column) and global $B-V$ colors (right column) for model 1 (top row) and model 2 (bottom row). The horizontal dotted lines delineate the range of observed values in blue LSB galaxies according to de Blok et al. (1995) and Zackrisson et al. (2005). The thick dashed lines show $E W H \alpha$ averaged over 1 Gyr. The arrow shows a (possible) reddening due to dust extinction for a fiducial value of the color excess $E(B-V)=0.1$ (McGaugh 1994).

5-6 Gyr. Both models begin to match the observed $E W(\mathrm{H} \alpha)$ only in the late evolution after $6 \mathrm{Gyr}$, with model 2 (Salpeter IMF) yielding a somewhat better fit. We note that all models feature short-term variations in $E W(\mathrm{H} \alpha)$ of such an amplitude that the corresponding equivalent widths may accidently go beyond either the upper or lower observed limits in the late evolution phase. This is however not alarming, since these fluctuations are short-lived and the observed range is inferred from just a few galaxies and may increase as more observational data become available.

Unlike model $E W(\mathrm{H} \alpha)$, there are no large short-term fluctuations in model $B-V$ colors shown in the right column of Fig. 10. Younger galaxies have on average bluer colors. The lowest observed color $B-V=0.3$ is reached after approximately 2.5-3.0 Gyr of evolution. Taking into account a possible reddening, the latter value may decrease to approximately 1.5-2.0 Gyr. A similar lower limit on the galactic age was inferred from numerical modelling of oxygen abundances in Sect. 6.1. However, model equivalent widths suggest a much larger value, 5-6 Gyr. This value may decrease slightly, if LSB galaxies feature truncated IMFs with a smaller upper mass limit, say $40 M_{\odot}$ instead of $100 M_{\odot}$. The oxygen abundance fluctuation spectrum $\mathcal{F}(A)$ can be used to better constrain the minimum age of blue LSB galaxies.

It is well known that blue LSB galaxies have $B-V$ colors that are systematically bluer than those of HSB galaxies (de Blok et al. 1995). It is tempting to assume that this fact may be indicative of blue LSB galaxies being systematically younger than their HSB counterparts. However, our evolved galaxies seem to have colors that fall within the observed limits. There is a slight possibility that the model $B-V$ color will exceed the upper observed limit if dust extinction is taken into account. But considering the uncertainty in dust content in blue LSB galaxies, this possibility is not conclusive. Even if blue LSB galaxies are younger than their HSB counterparts, the difference in age is not dramatic, of the order of a few Gyr at most. It is likely that blue LSB galaxies owe their bluer than usual colors to the sporadic nature of star formation (see e.g. McGaugh 1994; de Blok et al. 1995; Gerretsen \& de Blok 1999). 


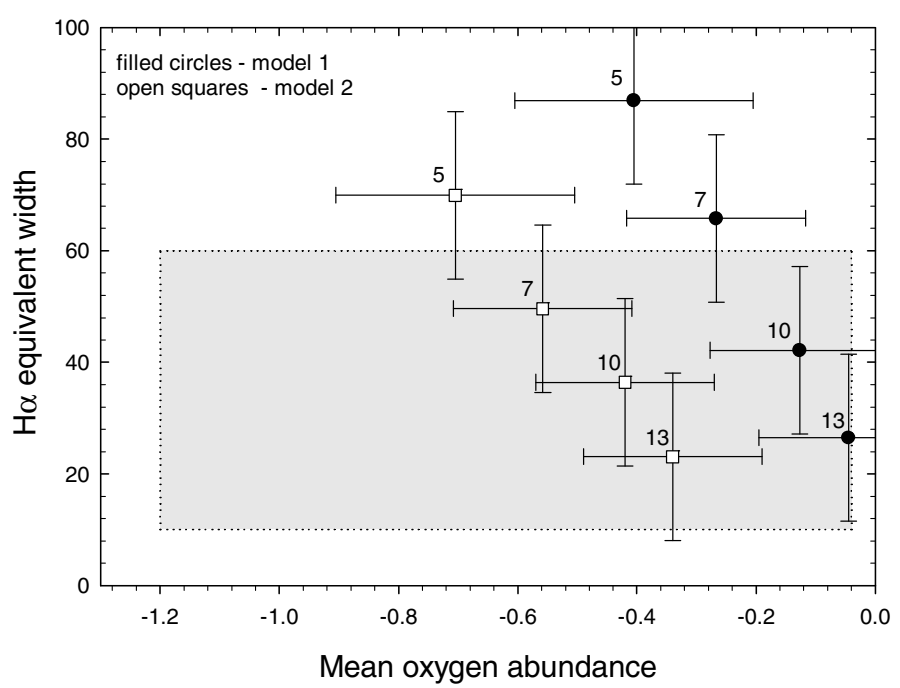

Fig. 11. Mean oxygen abundance versus $\mathrm{H} \alpha$ equivalent width. Each symbol presents a pair of data points corresponding to a model galaxy of specific age, as indicated in Gyr. Each symbol type corresponds to a particular numerical model specified in the upper-left corner. Bidirectional bars assigned to each symbol characterize a typical range of values found in our numerical modelling. The gray rectangular area delineates the range of observed values typical for blue LSB galaxies.

\subsection{Mean oxygen abundances versus $H \alpha$ equivalent widths}

Finally, we present the $\langle[\mathrm{O} / \mathrm{H}]\rangle-E W(\mathrm{H} \alpha)$ diagram in Fig. 11. Each symbol in this diagram represents a pair of data points $[\langle[\mathrm{O} / \mathrm{H}]\rangle, E W(\mathrm{H} \alpha)]$ corresponding to a specific galactic age in Gyr as indicated in the figure. In particular, filled circles and open squares represent models 1 and 2, respectively. Each symbol is assigned bi-directional bars, which characterize a typical range of values found in our numerical simulations. More specifically, the vertical bars illustrate typical short-term fluctuations of $E W(\mathrm{H} \alpha)$ within each model, while the horizontal bars represent a typical range of $\langle[\mathrm{O} / \mathrm{H}]\rangle$ found among models with identical IMFs but different SFRs. A gray rectangular area shows a typical range of observed oxygen abundances and $\mathrm{H} \alpha$ equivalent widths in blue LSB galaxies.

There are several important features in Fig. 11 that we want to emphasize. First, only those model galaxies that are older than 5-7 Gyr appear to fit into the observed range of both $E W(\mathrm{H} \alpha)$ and $B-V$. Younger galaxies fail to reproduce the observed $\mathrm{H} \alpha$ equivalent widths. Second, numerical models with standard mass limits $\left(m_{\text {low }}, m_{\text {up }}=0.1,100 M_{\odot}\right)$ populate the upper-right portion of the observed $E W(\mathrm{H} \alpha)$ vs. $B-V$ phase space. This suggests that at least some LSB galaxies may have IMFs that are different from those considered in the present work, i.e., they may have a truncated upper mass limit. Third, our modelling suggests an upper limit on the age of blue LSB galaxies that is comparable to or slightly less than the Hubble time. Forth, an accurate age prediction is possible if some independent knowledge of the IMF is available.

\section{Conclusions}

We have used numerical hydrodynamic modelling to study the long-term ( $\sim 13 \mathrm{Gyr})$ dynamical, chemical, and photometric evolution of blue LSB galaxies. Motivated by observational evidence, we have adopted a sporadic model for star formation, which utilizes highly localized, randomly distributed bursts of star formation that inject metals into the interstellar medium. We have considered several model galaxies that have masses and rotation curves typical for blue LSB galaxies and are characterized by distinct SFRs and IMFs. We look for chemical and photometric signatures (such as radial oxygen abundance fluctuations, time evolution of mean oxygen abundances, integrated $B-V$ colors and $\mathrm{H} \alpha$ equivalent widths) that can help to constrain the age of blue LSB galaxies. We find the following.

- Local bursts of star formation leave signatures in the disk in the form of oxygen enriched patches, which are subject to a continuing destructive influence of differential rotation, tidal forcing from spiral stellar density waves, and supernova shock waves from neighboring star formation sites. As a result, our model galaxy reveals short scale $(\sim 1-2 \mathrm{kpc})$ radial fluctuations in the oxygen abundance $[\mathrm{O} / \mathrm{H}]$. Typical amplitudes of these fluctuations are $0.5-1.0$ dex in (1-2) Gyr old galaxies but they decrease quickly to just $0.1-0.2$ dex in (5-13) Gyr old ones.

- The oxygen abundance fluctuation spectrum (i.e., the normalized number of fluctuations versus the fluctuation amplitude) has a characteristic shallow slope in (1-2) Gyr old galaxies. The fluctuation spectrum is age-dependent and steepens with time, reflecting the ongoing mixing of heavy elements in the disk. The dependence of the fluctuation spectrum on the model parameters and spatial resolution makes it possible to use the spectrum only for order-of-magnitude age estimates.

- The mean oxygen abundance $\langle[\mathrm{O} / \mathrm{H}]\rangle$ versus $E W(\mathrm{H} \alpha)$ diagram can be used to constrain the age of blue LSB galaxies, if some independent knowledge of the IMF is available.

- Numerical models with Salpeter and Kroupa IMFs and standard mass limits $\left(0.1-100 M_{\odot}\right)$ populate the upper-right portion of the observed $\langle[\mathrm{O} / \mathrm{H}]\rangle$ vs. $E W(\mathrm{H} \alpha)$ phase space. This implies that blue LSB galaxies are likely to have IMFs with a truncated upper mass limit.

- Our model data strongly suggest the existence of a minimum age for blue LSB galaxies. From model $B-V$ colors and mean oxygen abundances we infer a minimum age of 1.5-3.0 Gyr. However, model $\mathrm{H} \alpha$ equivalent widths imply a larger value, of the order of 5-6 Gyr. The latter value may decrease somewhat if LSB galaxies host IMFs with a truncated upper mass limit. A failure to observationally detect large oxygen abundance fluctuations, which, according to our modelling are typical for (1-2) Gyr old galaxies, will argue in favour of the more evolved nature of blue LSB galaxies.

- The oldest galaxies (13 Gyr) in all numerical models have mean oxygen abundances, colors and equivalent widths that lie within the observed range, suggesting that LSB galaxies are not considerably younger than their high surface brightness counterparts.

- Low metallicities and moderate cooling render the formation of cold gas with temperature $\$ 50 \mathrm{~K}$ inefficient. This fact implies that most of the cold phase may be in the form of atomic rather than molecular hydrogen, though further numerical simulations are needed to make firm conclusions.

- Sporadic star formation yields no radial abundance gradients in the disk of our model galaxy, in agreement with the lack of oxygen abundance gradients in blue LSB galaxies.

Acknowledgements. We appreciate the anonymous referee's suggestion to use population synthesis modelling. We thank Stacy McGaugh for allowing us to reanalyze his multicolor CCD image database of LSB galaxies. This work is partly supported by the Federal Agency of Education (project code RNP 2.1.1.3483 and 
2.1.1/1937), and by SFB 591. E.I.V. gratefully acknowledges support from an ACEnet Fellowship. The numerical simulations were performed on the Atlantic Computational Excellence Network (ACEnet). We also thank Professor Martin Houde for access to computational facilities.

\section{Appendix A: Disk scale height}

We derive the disk vertical scale height $Z$ at each computational cell via the equation of local vertical pressure balance

$\rho_{\mathrm{g}} c_{s}^{2}=2 \int_{0}^{Z} \rho_{\mathrm{g}}\left(g_{z}^{\text {gas }}+g_{z}^{\text {st }}+g_{z}^{\text {halo }}\right) \mathrm{d} z$,

where $\rho_{\mathrm{g}}$ is the gas volume density, $g_{z}^{\text {gas }}$ and $g_{z}^{\text {st }}$ are the vertical gravitational accelerations due to self-gravity of gas and stellar layers, respectively, and $g_{z}^{\text {halo }}$ is the gravitational pull of a dark matter halo. Assuming that $\rho_{\mathrm{g}}$ is a slowly varying function of vertical distance $z$ between $z=0$ (midplane) and $z=Z$ (i.e. $\left.\Sigma_{\mathrm{g}}=2 Z \rho_{\mathrm{g}}\right)$ and using the Gauss theorem, one can show that

$$
\begin{aligned}
& \int_{0}^{Z} \rho_{\mathrm{g}} g_{z}^{\mathrm{gas}} \mathrm{d} z=\frac{\pi}{4} G \Sigma_{\mathrm{g}}^{2} \\
& \int_{0}^{Z} \rho_{\mathrm{g}} g_{z}^{\mathrm{st}} \mathrm{d} z=\frac{\pi}{4} G \Sigma_{\mathrm{st}} \Sigma_{\mathrm{g}} \\
& \int_{0}^{Z} \rho_{\mathrm{g}} g_{z}^{\text {halo }} \mathrm{d} z=\frac{G M_{\mathrm{h}}(r) \rho_{\mathrm{g}}}{r}\left\{1-\left[1+\left(\frac{\Sigma_{\mathrm{g}}}{2 \rho_{\mathrm{g}} r}\right)\right]^{-1 / 2}\right\},
\end{aligned}
$$

where $r$ is the radial distance and $M_{h}(r)$ is the mass of the dark matter halo located inside radius $r$. In Eq. (A.3) we have assumed that the volume density of stars can be written as $\Sigma_{\text {st }} / Z$. Substituting Eqs. (A.2)-(A.4) back into Eq. (A.1) we obtain

$$
\begin{aligned}
\rho_{\mathrm{g}} c_{\mathrm{s}}^{2}= & \frac{\pi}{2} G \Sigma_{\mathrm{g}}+\frac{\pi}{2} G \Sigma_{\mathrm{g}} \Sigma_{\mathrm{st}} \\
& +\frac{2 G M_{\mathrm{h}}(r) \rho_{\mathrm{g}}}{r}\left\{1-\left[1+\left(\frac{\Sigma_{\mathrm{g}}}{2 \rho_{\mathrm{g}} r}\right)\right]^{-1 / 2}\right\} .
\end{aligned}
$$

This can be solved for $\rho_{\mathrm{g}}$ given the model's known $c_{\mathrm{s}}^{2}, \Sigma_{\mathrm{g}}, \Sigma_{\mathrm{st}}$, and $M_{\mathrm{h}}(r)$. The vertical scale height is finally derived as $Z=$ $\Sigma_{\mathrm{g}} /\left(2 \rho_{\mathrm{g}}\right)$.

\section{Appendix B: Population synthesis modelling}

We make use of the single-burst models by Bruzual \& Charlot (2003) to synthesize broad-band colors and $\mathrm{H} \alpha$ equivalent widths. The continuum level $F_{\mathrm{c}}(t)$ near the $\mathrm{H} \alpha$ line at time $t$ can be found as

$F_{\mathrm{c}}(t)=\int_{0}^{t} f_{\mathrm{c}}\left(t-\tau, Z_{m}\right) \Psi(\tau) \mathrm{d} \tau$,

where $\Psi(\tau)$ is the SFR at time $\tau$, and $f_{\mathrm{c}}\left(t-\tau, Z_{\mathrm{m}}\right)$ is the continuum luminosity per wavelength for a single-burst stellar population with age $t-\tau$ and metallicity $Z_{\mathrm{m}}$.

The $\mathrm{H} \alpha$ luminosity $L_{\mathrm{H} \alpha}(t)$ at time $t$ is estimated as $L_{\mathrm{H} \alpha}[\mathrm{erg} / \mathrm{s}]=1.36 \times 10^{-12} N_{\mathrm{Lyc}}[1 / \mathrm{s}]$ (Kennicutt 1983), where $N_{\text {Lyc }}$ is the rate of ionizing photon production derived directly from Bruzual and Charlot's population synthesis modelling. The resulting equivalent width is found as $E W(\mathrm{H} \alpha)=L_{\mathrm{H} \alpha} / F_{\mathrm{c}}$. The broad-band $B-V$ color at time $t$ is found via $B$ and

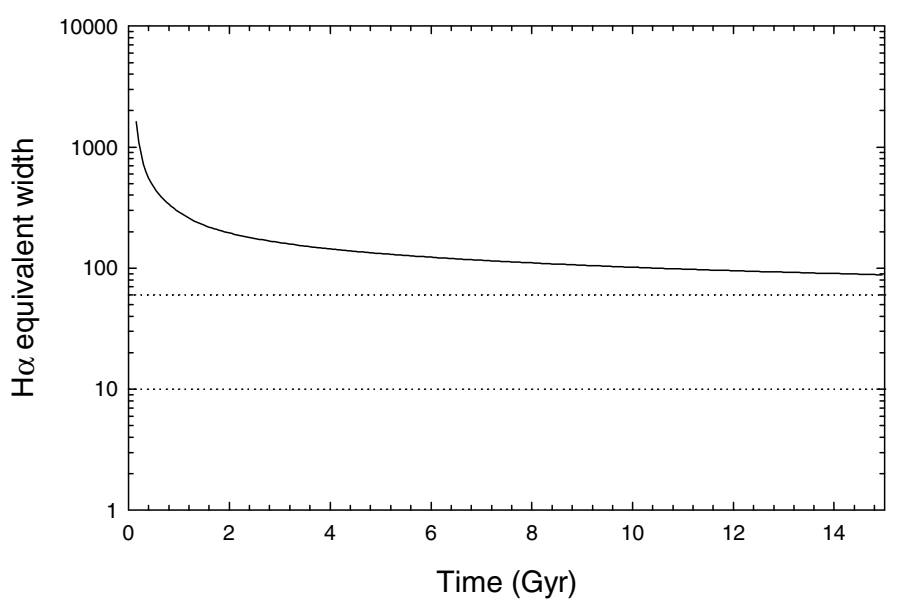

Fig. B.1. $\mathrm{H} \alpha$ equivalent width versus galactic age for a test model as described in the text. The solid line presents the data for metallicity $Z_{\mathrm{m}}=0.001$. The horizontal dotted lines delineate the observed range of values for blue LSB galaxies.

$V$ luminosities $\left(L_{B}(t)\right.$ and $L_{V}(t)$, respectively) calculated using Eq. (B.1), in which $f_{\mathrm{c}}$ is replaced with luminosities in the $B$ and $V$ bands, respectively. The resulting color is $(B-V)=$ $-2.5 \log _{10}\left[L_{B}(t) / L_{V}(t)\right]$.

We perform a consistency test on our population synthesis model by comparing our predicted $E W(\mathrm{H} \alpha)$ with those of Zackrisson et al. (2005) for a model galaxy with a constant (over 15 Gyr) SFR, total mass of formed stars $10^{10} M_{\odot}$, metallicity $Z_{\mathrm{m}}=0.001$, and Salpeter IMF with mass limits $\left(m_{\mathrm{low}}, m_{\mathrm{up}}\right)=$ $(0.1,120) M_{\odot}$. The resulting $\mathrm{H} \alpha$ equivalent width as a function of time is shown in Fig. B.1 by the solid line. Our model $\mathrm{H} \alpha$ equivalent widths match those of Zackrisson et al. (2005) quite well - at $t=15 \mathrm{Gyr}$ we find $E W(\mathrm{H} \alpha)=88 \AA$, whereas Fig. 2 in Zackrisson et al. (2005) shows $E W(\mathrm{H} \alpha) \approx 86 \AA$. This example demonstrates that a constant SFR produces too large $\mathrm{H} \alpha$ equivalent widths and hence is unlikely.

Finally, we would like to note that $Z_{\mathrm{m}}=0.001$ (or $[\mathrm{O} / \mathrm{H}]=-1.3$ ) is not typical for present-day blue LSB galaxies. According to de Blok \& van der Hulst (1998) and Kuzio de Narray et al. (2004), the mean oxygen abundance of blue LSB galaxies is $\langle[\mathrm{O} / \mathrm{H}]\rangle_{\mathrm{LSB}}=-0.85$ dex or one seventh of the solar metallicity. Higher metallicity is expected to yield lower $\mathrm{H} \alpha$ equivalent widths.

\section{References}

Auld, R., de Blok, W. J. G., Bell, E., \& Davies, J. I. 2006, MNRAS, 366, 1475 Bomans, D. J., Chu, Y.-H., \& Hopp, U. 1997, AJ, 113, 1678 Bruzual, G., \& Charlot, S. 2003, MNRAS, 344, 1000 de Avillez, M. A., \& MacLow, M.-M. 2002, ApJ, 581, 1047 de Avillez, M. A., \& Breitschwerdt, D. 2005, ApJ, 634, L65 de Blok, W. J. G., \& van der Hulst, J. M. 1998, A\&A, 335, 421 de Blok, W. J. G., van der Hulst, J. M., \& Bothun, G. D. 1995, MNRAS, 274, 235

de Blok, W. J. G., McGaugh, S. S., \& van der Hulst, J. M. 1996, MNRAS, 283, 18 Draine, B. T., \& Salpeter, E. E. 1979, ApJ, 231,

Gerretsen, J. P. E., \& de Blok, W. J. G. 1999, A\&A, 342, 655

Haberzettl, L., Bomans, D. J., \& Dettmar, R.-J. 2005, in The Evolution of Starbursts: The 331st Wilhelm and Else Heraeus Seminar. AIP Conf. Proc., 783, 296

Jimenez, R., Padoan, P., Matteucci F., \& Heavens, A. F. 1998, MNRAS, 299, 123 Kennicutt, R. 1983, ApJ, 272, 54

Kennicutt, R. C. 1998, ARA\&A, 36, 189

Köppen, J., \& Arimoto, N. 1991, A\&AS, 87, 109 
Kroupa, P. 2001, MNRAS, 322, 231

Kunth, D., \& Sargent, W. L. W. 1986, ApJ, 300, 496

Kuzio de Naray, R., McGaugh, S. S., \& de Blok, W. J. G. 2004, MNRAS, 355, 887

Kuzio de Naray, R., McGaugh, S. S., deBlok, W. J. G., \& Bosma, A. 2006, ApJS, 165,461

Martin, C. L., \& Kennicutt, R. C. 2001, ApJ, 555, 301

McGaugh, S. S. 1994, ApJ, 426, 135

McGaugh, S. S. \& Bothun, G. D. 1994, AJ, 107, 530

McGaugh, S. S., Bothun, G. D., \& Schombert, J. M. 1995a, AJ, 110, 573

McGaugh, S. S., Schombert, J. M., \& Bothun, G. D. 1995b, AJ, 109, 2019

McGaugh, S. S., \& de Blok, W. J. G. 1997, ApJ, 481, 689

Mihos, J. C., Spaans, M., \& McGaugh, S. S. 1999, ApJ, 515, 89

O’Neil, K., \& Bothun, G. 2000, ApJ, 529, 811

O’Neil, K., Bothun, G. D., Schombert, J., Cornell, M. E., \& Impey, C. D. 1997, AJ, 144, 244

Padoan, P., Jimenez, R., \& Antonuccio-Delogu, V. 1997, ApJ, 481, L27

Palla, F., \& Stachler, S. W. 2002, ApJ, 581, 1194
Pickering, T. E. 1999, in The Low Surface Brightness Universe, ed. J. I., Davies, C., Impey, \& S., Phillipps, ASP Conf. Ser., 170, 214

Rönnback, J., \& Bergvall, N. 1995, A\&A, 302, 353

Scalo, J., \& Elmegreen, B. G., ARA\&A, 42, 275

Schombert, J. M., McCaugh, S. S., \& Eder, J. A. 2001, AJ, 121, 2420

Sprayberry, D., Impey, C. D., Irwin, M. J., \& Bothn, G. D. 1997, ApJ, 481, 104

Thomas, D., Maraston, C., Bender, R., \& Mendes de Oliveira, C. 2005, ApJ, 621, 673

Trachternach, C., Bomans, D., Haberzettl, L., \& Dettmar, R.-J. 2006, A\&A, 458, 341

van den Hoek, L. B., de Blok, W. J. G., van der Hulst, J. M., \& de Jong, T. 2000, A\&AS, 357, 397

Vorobyov, E. I. 2003, A\&A, 407, 913

Vorobyov, E. I., \& Shchekinov, Yu. A. 2005, New Astron., 11, 240

Vorobyov, E. I., \& Basu, S. 2006, ApJ, 650, 956

Wada, K., \& Norman, C. A. 2001, ApJ, 547, 172

Woosley, S. E., \& Weaver, T. A. 1995, ApJS, 101, 181

Zackrisson, E., Bergvall, N., \& Östlin, G. 2005, A\&A, 435, 29 